\title{
BACTERIOPHAGE TYPING OF ENTERIC PATHOGENS AND STAPHYLOCOCCI AND ITS USE IN EPIDEMIOLOGY
}

\author{
BY \\ E. S. ANDERSON AND R. E. O. WILLIAMS \\ From the Central Enteric Reference Laboratory and Bureau and the Staphylococcus Reference Laboratory, \\ Public Health Laboratory Service, Colindale, London, N.W.9
}

(RECEIVED FOR PUBLICATION APRIL 6, 1956)

One of the main problems that have beset the epidemiologist since the earliest days of bacteriology is that of proving that all strains of a particular pathogenic organism isolated during the course of an outbreak originate from a common source. This proof is needed not only for the cultures isolated from patients, but also for those isolated from vehicles of infection such as contaminated food or fomites, and from carriersanimal or human-who may have been the original source of infection. The evolution of much of bacteriology has been determined by expediency, and this need to recognize particular epidemic strains of pathogenic organisms has encouraged a great deal of study of methods of identification.

So far as diagnostic bacteriology is concerned, it is now accepted that particular epidemic diseases are caused by single species of bacteria or by several species of a genus. The production of enteric fever by typhoid and paratyphoid bacilli is an example of different members of one bacteriological genus, Salmonella, causing a similar disease. The division into species of the larger groups of organisms, such as the haemolytic streptococci, the clostridia, and the dysentery bacilli, has often shown that the members even of the finer taxonomic divisions are responsible for characteristic infections. Each such step in identification has brought us nearer to the goal of the epidemiologist, which is the acquisition of a method of distinguishing different strains of a single bacterial species.

If, in a general way, "species" of pathogenic organisms are thought of as groups that produce characteristic reactions in their hosts, the term "type" may be used for the varieties that are distinguishable within the species by laboratory methods only. With any organism that is wide- spread, and especially for an organism that is commonly carried by healthy persons, the recognition of types is essential for any adequate epidemiological study.

In different species and at different times, various methods of recognizing types have been developed. Thus Kristensen and Henriksen (1926) and Kristensen (1938) attempted to subdivide the typhoid bacillus, using the differential fermentation of L-arabinose and xylose as the criterion. Kristensen's method distinguishes three biochemical types of Salmonella typhi. Type I ferments xylose but not arabinose; type II ferments neither xylose nor arabinose; and type III ferments both sugars. As this scheme distinguishes so few types, and as most strains of $S$. typhi belong to type I, the method is inadequate for the recognition of epidemic strains.

Serological methods of recognizing types have also been widely used, and in, for example, the Group A streptococci, have proved very helpful. Serological typing has also been used for staphylococci but has hitherto generally been unsatisfactory, again because too few types were recognizable.

In 1938 Craigie and Yen announced their Viphage typing scheme for $S$. typhi. This was soon established as the method of choice for the epidemiological "fingerprinting" of typhoid strains. It has also served as a model for the development of all subsequent schemes for the typing of organisms by means of bacteriophage. Later work has shown that phages are often the most effective tools for the classification of bacteria at the intraspecific level. Phage typing schemes are now used in this and other countries for S. typhi, S. paratyphi B, S. typhimurium, Shigella sonnei, and Staphylococcus aureus. 


\section{REQUIREMENTS OF A TYPING METHOD}

In order to be of practical value any typing scheme should have the following features:

(1) The types must be stable. If it is impossible to be certain that a given type will exhibit the same reactions from week to week, the method is of very limited value. On the other hand, certain variations in particular typing reactions may be constant and, if they are easily recognized, may serve to define subtypes or "variations" of types.

(2) Ideally there should be an adequate number of types, but not too many. When a scheme subdivides a species into only three or four types, the epidemiological information it can give is limited, especially if one type is very common. When a method yields a very large number of types one may suspect that subdivision is being attempted on too fine a basis, and the epidemiological validity of the method needs to be studied especially carefully.

(3) The technique should be reasonably simple and give clear-cut and reproducible results. Reproducibility is difficult to attain with a method that relies on small quantitative differences in the reactions. With phage typing methods in particular, type distinctions that depend on small differences in the degree of lysis by one phage are useless because the number of plaques developing in a phage-inoculated area is influenced by environmental factors, and the same phage inoculum may yield widely different plaque counts on the same strain on different occasions due to minor variations of medium, temperature, etc., that are impossible to control. Without doubt it is best if one can rely on the distinction between positive and negative reactions.

(4) The typing reagents should be stable.

(5) The method should be capable of standardization; the typing reagents should all be derived initially at least from a common source, and the technical methods and interpretation of results should be generally agreed upon.

(6) The results of the test should be available quickly.

(7) Before being accepted for general use any typing scheme must be subjected to rigorous epidemiological tests in order that its reliability may be established.

The Vi-phage typing scheme for the typhoid bacillus conforms most closely with all these requirements: in stability of type, in the convenient number of types recognized, and in the readiness with which it can be standardized and brought to a very high degree of accuracy and reproducibility. The typing of $S$. paratyphi B is only slightly less satisfactory, mainly on account of the lower specificity of the typing phages. For both these systems stocks of phage are prepared in one centre and distributed to laboratories throughout the world. In contrast, the typing scheme for staphylococci suffers from the relative non-specificity of the typing phages; this entails undue reliance on lytic patterns, often difficult to interpret, for characterizing the strains. The number of patterns recognizable is also too large for convenience, but this is not all loss, because the staphylococcus is so ubiquitous an organism that fine distinctions between strains are, when they can be shown to be stable, very useful.

\section{TERMINOLOGY}

As a number of the terms to be used in the following article may be unfamiliar to the clinical pathologist, it may be better to define them.

A lysogenic bacterial strain is one in which every cell is endowed with the ability to produce bacteriophage and to transmit this property to its progeny. Lysogenic strains show no visible signs of phage infection and are resistant to the phages they carry. Phages carried in this way are called temperate phages (Jacob, Lwoff, Siminovitch, and Wollman, 1953). They can only be detected by their ability to lyse other strains which are known as indicator strains.

Lysogenization is the production of the lysogenic state. It follows the attack of a temperate phage on an indicator strain; many of the host cells are destroyed, but a proportion become lysogenic. There is no indication that lysogeny acquired in this way differs from that encountered under natural conditions.

Prophage is the latent state in which phage exists in and is transmitted by lysogenic bacteria.

Virulent phage is one that cannot enter into a lysogenic relationship with host cells. Thus, the only cells surviving its attack are resistant to it for some special reason, such as inability to absorb it.

Phage type, Vi-phage type, Vi-type, type are expressions used to describe the types of organism distinguishable by phages.

A bacterial phage type is lysed by its homologous typing phage, but may be resistant to a heterologous phage.

A host-range mutant of a phage possesses the power to attack strains that are resistant to the parent phage. 
A type-determining phage is a temperate phage the presence of which in the cell exercises a partial control over the sensitivity of a bacterial phage type to a range of typing phages.

For further information concerning the lysogenic state the reader is referred to the excellent review by Lwoff (1953).

\section{ENTERIC PHAGE TYPING Salmonella typhi}

Soon after Felix and Pitt (1934) published their description of the Vi antigen of S. typhi, a number of workers (Craigie and Brandon, 1936; Scholtens, 1936; Sertic and Boulgakov, 1936) discovered bacteriophages that were specific for the Vipositive form of the organism. These Vi phages, as they are now called, would attack only organisms possessing the $\mathrm{Vi}$ antigen, and were not adsorbed to the bacterial cells unless this antigen was present. In 1938 Craigie and Yen (1938a, b) described four new virulent Vi phages which they designated I, II, III, and IV. These phages differed in physical properties and were serologically distinct from each other. Craigie and Yen had originally intended to evolve a typing scheme for the typhoid bacillus based on the differential reactions of different strains of the organism to the four phages. However, the peculiar behaviour of one of them-Vi-phage II-led them to focus special attention on it. This phage proved to have the unusual property of adapting itself to strains of $S$. typhi in such a way that it became highly specific for the last strain on which it had been propagated. Craigie and Yen found that any adaptation of Vi-phage II would lyse strains similar to that on which it had last been grown, and by successive adaptations of the $\mathrm{Vi}$ phage they were able to subdivide a collection of typhoid strains into 11 types. It was shown that epidemiologically related strains were always lysed by the same adapted phage, and Craigie and Yen's scheme formed the nucleus of the Vi-phage typing scheme for $S$. typhi that is in widespread use at the present time. The potentialities of the method were quickly realized by the late Dr. A. Felix, and its development, extension, and internationalization are due in large part to his work (see Felix, 1943, 1944, 1950, 1951, 1955).

There are at present 33 internationally recognized Vi-types of the typhoid bacillus distinguishable by specifically adapted preparations of Vi-phage II. The reactions of these types with their typing preparations in routine test dilution are shown in Table $\mathbf{I}$.

Type $\mathbf{A}$ is sensitive to all adaptations of $\mathrm{Vi}$ phage II irrespective of their specificity. Some types react with more than one phage, and, as $\underset{\vec{*}}{\vec{*}}$ Table I shows, groups have been created on the basis of such cross-reactions, which are in some instances due to known relations between certain types.

Use of the Routine Test Dilution of Typing $\frac{\bar{\sigma}}{\vec{D}}$ Phages.-One of the most important principles $\stackrel{\mathbb{Q}}{\circ}$ that have emerged in the systems of phage typing $\%$ used at present is that of employing phages in $\overrightarrow{0}$ routine test dilution (R.T.D.) or, as Craigie and Yen (1938) called it, the critical test dilution. The $\vec{\omega}$ R.T.D. of a phage is the highest dilution which $\stackrel{\circ}{\circ}$ produces confluent or semi-confluent lysis on its homologous type strain, that is, the type on which the phage has been propagated. The conception 0 arose from the Vi-phage typing scheme of the typhoid bacillus. Any of the adapted Vi-typing phage preparations will produce the appearance of $\vec{z}$ confluent lysis when applied undiluted to any of the Vi-types of $S$. typhi. This is due to the fact that all Vi-types of $S$. typhi will adsorb all the $\mathscr{E}$ typing adaptations of Vi-phage II, irrespective of their specificity (Craigie, 1940). It has been shown recently (Anderson and Fraser, 1956) that this adsorption is lethal to the bacteria. although $\overline{0}$ multiplication of the phage with lysis of the host cells may not occur. When the phage is applied $\stackrel{\square}{\AA}$ in sufficiently high concentration, massive cell $\overrightarrow{\vec{O}}$ destruction results in the affected area. This pro- 3 duces the appearance of confluent lysis and is often indistinguishable from the specific lysis caused by the action of a typing phage on its homologous type strain. Moreover, a concentrated lysate con- tains mutant particles able to lyse many Vi-types 3 of the typhoid bacillus. Each such particle may produce a plaque and, if a sufficiently large number of them are present, confluent lysis of the strain to 0 which the phage is applied will result. For these reasons, it is unsound to apply the phages undiluted to the organism under test. Each specific preparation is used in the highest possible dilution 0 consonant with the production of satisfactory lysis N on its type strain. At this level of phage dilution the occurrence of cross-reactions is reduced to a minimum and each type of organism reveals its specificity.

This principle is now applied to most of the phage typing systems in current use. As will be 7 seen later, none of the phages used for the para- 0

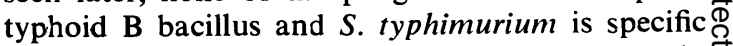

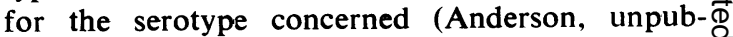
lished ; Cherry, Davis, and Edwards, 1953 ; Sicca and D'Amelio, 1953). Undiluted preparations of these phages contain a wide variety of host-range 


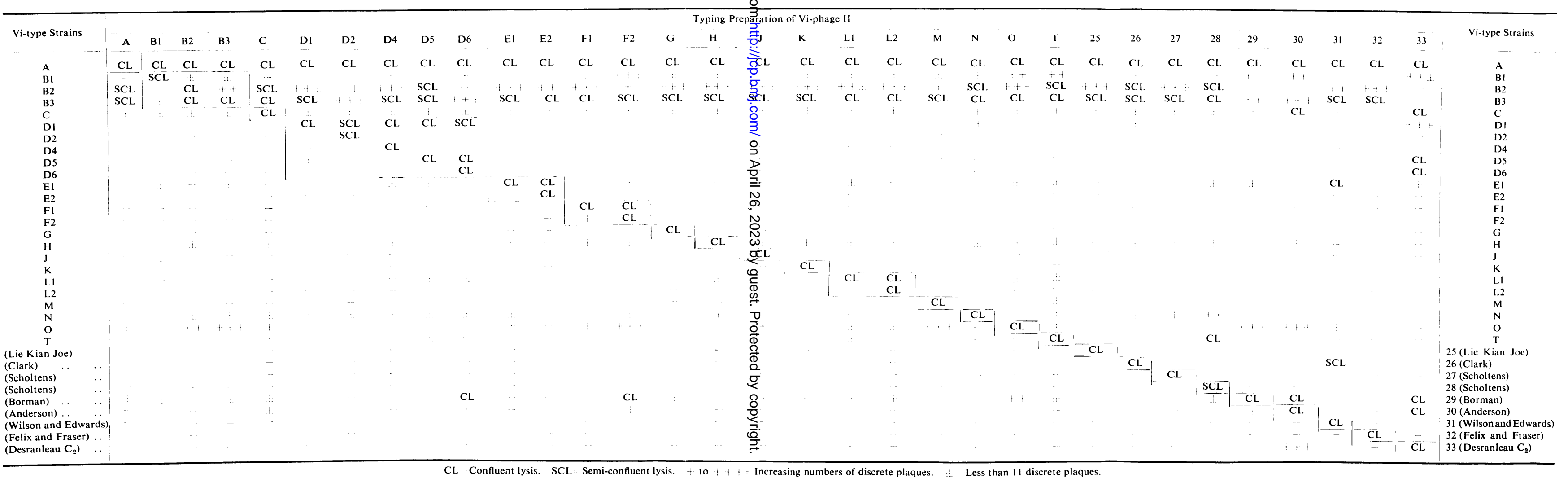


mutants that will lyse many salmonella serotypes. Thus, it is again important to approach specificity by using the phages in the highest possible dilution. In the case of all phage typing systems except that of $S$. typhi, there is an additional necessity which arises out of the fact that, even in R.T.D., the phages are not really serotype specific. Thus, some types of $S$. typhimurium are sensitive to the paratyphoid B typing phages. This makes it essential to establish characteristic pattern reactions for the different phage types of the particular serotype for which a typing scheme is being employed.

The employment of the R.T.D. and, in the case of salmonellas other than S. typhi, the establishment of type specific pattern reactions, render diagnosis a reasonably simple matter with the commoner phage types.

It may be added here that the specificity of these phage-reaction patterns is such that they can be used to identify salmonella serotypes. This is valuable when a salmonella strain presents a partial flagellar antigenic deficiency, or is non-motile, and cannot, therefore, be typed serologically (Anderson, 1955b).

Preparation of Routine Typing Phages for Distribution.--Provided that the prescribed technique is adhered to in the routine use of enteric phage typing ieagents the results are comparable wherever the method is used. With this reduction of technical variations to a minimum, a true picture of the distribution throughout the world of the phage types of $S$. typhi and $S$. paratyphi $\mathrm{B}$ has been obtained. In order to maintain the low level of variables, the principle has long been accepted by the International Committee for Enteric Phage Typing (I.C.E.P.T.) that the typing phages prepared by one centre should be used by all workers.

The preparation of typhoid Vi-typing phages will be described in some detail. The same principleswith necessary modifications-are followed in the preparation of typing phages for the paratyphoid $B$ bacillus and $S$. typhimurium. The various techniques described here are those of the Central Enteric Reference Laboratory (C.E.R.L.), Colindale.

All the Vi-typing phages in general use are derived from phage A of Craigie and Yen (1938), which is the "wild" type of Vi-phage II, and the majority are still propagated on the type strains of $S$. typhi to which they were originally adapted. These phages have been purified by repeated single-plaque isolation, and, because of the precautions that have been taken to maintain their purity, they can be grown in bulk from stock lysates. Experience has shown that an approximately equal concentration of phage particles and host cells usually yields the highest titre phages. As a routine, $1.5 \times 10^{9}$ cells of the correct type in the logarithmic phase of growth are inoculated into
$150 \mathrm{ml}$. of pre-warmed Difco broth in screw-capped bottles together with about the same number of particles of the homologous typing phage. The bottles are incubated at $38.5^{\circ} \mathrm{C}$. for as long as lysis continues, to a maximum of about seven and a half hours. They are then heated at $57^{\circ} \mathrm{C}$. for 40 minutes to kill the host cells, and the lysates are centrifuged to remove the dead bacteria. A preliminary titration of the phage is carried out to determine its R.T.D. This titration is performed on type $A$, which is sensitive to all adaptations of Vi-phage II, and on the homologous Vi-type strain of $S$. typhi on which the phage has been propagated. Although it is always hoped to obtain an R.T.D. that gives confluent lysis, this ideal may have to be abandoned when the phage produces very small plaques on its indicator strain. However, nothing less than semiconfluent lysis is accepted as a usable R.T.D.

It is sometimes impossible to prepare a phage of sufficiently high titre in liquid medium, whatever variations in phage and cell inocula or time of incubation may be tried. Under such conditions it has often been possible to obtain high-titre phages by using the agarlayer technique currently employed in much academic phage research. The phage inoculum is added to a suspension of cells of the requisite Vi-type in $3.0 \mathrm{ml}$. of a $0.45 \%$ Davis New Zealand powdered agar in Difco broth. The mixture is poured on to a basal layer of Difco agar of the usual composition in a $9 \mathrm{~cm}$. Petri dish. The cell inoculum is about $4.0 \times$ $10^{8}$ organisms, and the phage inoculum is the smallest number of particles that will yield confluent lysis. This ensures that the maximum number of cycles of phage growth will occur in the plate and that the minimum number of susceptible cells will be left to adsorb the phage during harvesting. The plates are incubated for 16 hours. The phages are harvested by flooding the plates with $6 \mathrm{ml}$. of Difco broth and scraping off the surface layer of soft agar with a platinum knife. The mixture of broth and agar pulp is gently sucked in and out of a wide-mouthed 10-ml. pipette a few times and transferred to 20-ml. bottles, which are heated at $57^{\circ} \mathrm{C}$. for 40 minutes to kill the typhoid bacilli and centrifuged as before. Using this method phage A suspensions with titres exceeding $10^{12}$ per ml. have been obtained-about 100 times stronger than in fluid medium. Phages difficult to grow in nutrient broth usually give a reasonable R.T.D. in agar layer. The R.T.D. is determined by exactly the same method as that used for phage grown in liquid medium.

After its R.T.D. has been ascertained the newly grown phage is tested in R.T.D. and in 100 times this strength on the full set of Vi-phage strains of S. typhi. It is also tested in much higher concentrations on $S$. typhi strain 0901 which, as it is a Vi-negative strain, is resistant to all the Vi-typing phages ; this strain is used to detect contaminant non- $\mathrm{Vi}$ phages in the lysate. It may be added here that such contamination is extremely rare, but the Vi-typing preparations grown on types carrying heat-stable determining phages (see section on type specificity) contain these 
determining phages in low titre. Fortunately, the R.T.D. of the Vi-typing preparations is usually well beyond the extinction titre of the determining phages so that the latter do not present a source of error in routine phage typing.

The typhoid Vi-typing phages are stable for very long periods at $4^{\circ} \mathrm{C}$. Concentrated preparations maintain their titre and specificity for at least 10 years at this temperature. The phages distributed at 100 times the strength of the R.T.D. show a similar degree of stability and the routine test dilution of these phages is certainly stable for many months at $4^{\circ} \mathrm{C}$. The $S$. paratyphi B and S. typhimurium typing phages are also stable at $4^{\circ} \mathrm{C}$. Most of the Vi-type strains of $S$. typhi are stable for many years when maintained at room temperature on dry Dorset egg slopes.

Technique of Enteric Phage Typing.-The technique is essentially that developed by Craigie and Felix (1947). A closely similar technique is followed in this work in all other countries using the method.

Medium.-Bacto dehydrated nutrient broth (Difco Laboratories) is used for routine typing. The liquid medium, which is hereafter referred to as Difco broth. has the following formula:

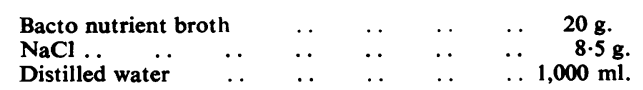

The medium is autoclaved at $15 \mathrm{lb}$. for 25 minutes. The final $p \mathrm{H}$ is 6.8 .

For the preparation of solid medium (Difco agar) $1.3 \%$ Davis New Zealand powdered agar is added.

Cultures to be phage typed are inoculated into $2 \mathrm{ml}$. of Difco broth to give a barely visible turbidity and incubated with agitation at $38.5^{\circ} \mathrm{C}$. until the opacity is equivalent to approximately $5 \times 10^{8}$ organisms $/ \mathrm{ml}$. ; this turbidity is reached in about two and a half hours. Petri dishes of $9 \mathrm{~cm}$. diameter are used, each containing about $20 \mathrm{ml}$. of Difco agar ; before use these are incubated overnight with the lid closed and for a further hour with the lid open in order to dry the agar surface. Fig. 1 shows the manner in which plates are marked for phage typing Salmonella typhi. As many phage types of $S$. typhi are rare, it is convenient to use pools of a number of the phages.

Phage $A$ is the most specific of the adaptations of Vi-phage II ; when used in R.T.D., it produces confluent lysis only on type $A$.

Some cultures become more sensitive to phage A when they are in the process of losing Vi-type specificity (Anderson and Felix, 1953a). This process will be discussed more fully later ; it is sufficient here to indicate that an idea of the extent of the "degradation," as it is called, can be gained from the reaction of a culture to phage A applied 20 times stronger than the R.T.D. The dilution is referred to as $\mathrm{A}(1.500)$. As Fig. 1 shows, the following spots of phage are used in the C.E.R.L.: A (1,500), A (R.T.D.), $\left[B_{1}, B_{2}, B_{3}\right], C, D_{1},\left[D_{2}, D_{4}, D_{5}, D_{6}\right], E_{1}, E_{2}, F_{1}, F_{2}$, [G, H, K], J, [L $\left.\mathrm{L}_{1} \mathrm{~L}_{2}\right], \mathrm{M},[\mathrm{N}, \mathrm{O}, \mathrm{T}],[25,26,27,28$, 29], [30, 31, 32, 33], [Vi-phages I and IV], Vi-phage III, [Vi-phages $V$ and VI], [anti-O]. The phages en-

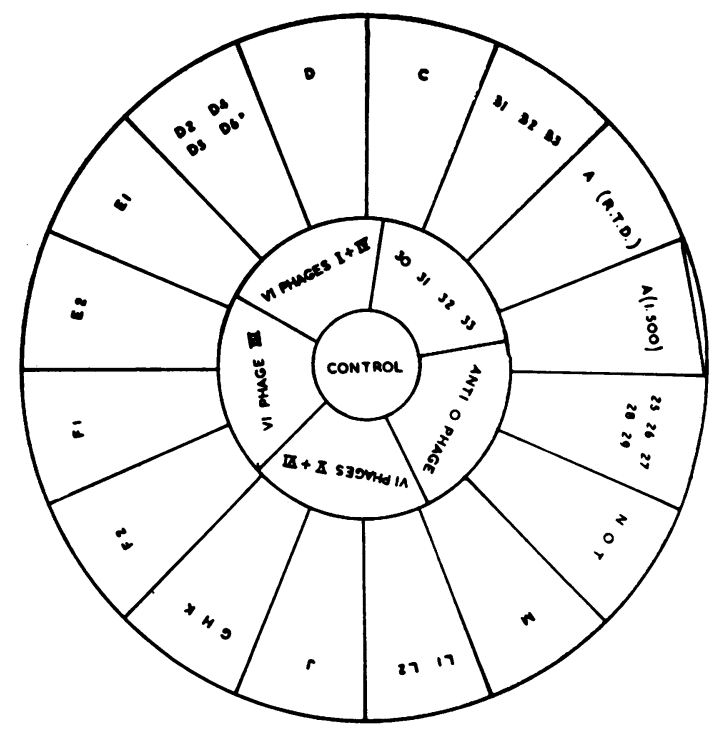

FIG. 1.-Method of marking plates for Vi-phage typing of $S$. typhi.

closed in square brackets are pooled. The range of adapted phages has now been increased to cover a further 12 recently identified Vi-types. Vi-phages I, III, and IV were described by Craigie and Yen (1938). Vi-phages V and VI were discovered by Desranleau and Martin (1950). The phage designated "anti-O" consists of a pool of three closely related phages which were described by Felix and Callow (1943). They were considered to be " $O$ " phages because they attacked the non-Vi form of the typhoid bacillus and also lysed many other salmonellas. Moreover, they also lyse a number of rough strains, and would be better designated " non-Vi" phages. Vi-phages I, III. IV, $\mathrm{V}$, and VI and the anti-O phage are unadapted. They serve a double purpose. If a culture does not show an identifiable type reaction with the adaptations of Vi-phage II it may, nevertheless, give a distinctive reaction pattern with the unadapted $\mathrm{Vi}$ - and anti-O phages. This is often sufficient to characterize a strain and may be as useful epidemiologically as a specific type-designation. Secondly, the Vi-phages when used in R.T.D. are specific for the typhoid bacillus, so that lysis by any of them indicates that the culture under examination is $S$. typhi.

The same technique of setting up typing plates is used for S. typhi, S. paratyphi B, and S. typhimurium. Standard loops of $3.0 \mathrm{~mm}$. internal diameter are used throughout, and in experienced hands these deliver a volume very close to $0.01 \mathrm{ml}$. One loopful of the incubated broth culture of the strain under examination is distributed into each of the various sections of the plate and evenly spread into circles about $1 \mathrm{~cm}$. in diameter. When the inoculated areas are dry, the typing phages are spotted on to them with a loop of the same size. The phages are not spread; they form a visible central zone on the bacterial inocula even 
before incubation. After the drops of phage have dried, the plates are incubated at $38.5^{\circ} \mathrm{C}$. First readings are taken after seven hours with $S$. typhi and after five hours with $S$. paratyphi B and $S$. typhimurium. A second reading is taken when the total incubation is 24 hours. It is often possible to report findings after the first reading, and in cases of urgency this can be done by telegram or telephone on the day of receipt of the culture.

Reading and Interpretation of Results.-Readings are carried out with a $\times 10$ aplanat hand lens through the bottom of the plates, using both direct and oblique illumination. All-glass Petri dishes are used, so that the lids need not be removed for readings. The various degrees of lysis are recorded as shown in Table II.

TABLE II

METHOD OF RECORDING DEGREES OF LYSIS ON ENTERIC PHAGE-TYPING PLATES

\begin{tabular}{|c|c|c|c|}
\hline Plaque Sizes & \multicolumn{2}{|c|}{ Plaque Numbers } & Lysis \\
\hline $\begin{array}{l}\mathbf{L}=\text { large } \\
\mathbf{N}=\text { normal } \\
\mathbf{S}=\text { small } \\
\quad=\text { minute, } \\
\text { visible only } \\
\text { with hand } \\
\text { lens } \\
\mu=\text { micro }\end{array}$ & $\begin{aligned} & 0 \text { to } 5 \mathrm{pl} \\
\pm & =6-20 \\
+ & =21-40 \\
+ \pm & =41-60 \\
++ & =61-80 \\
++t & =81-120 \\
+++ & =>120\end{aligned}$ & $\begin{array}{l}\text { aques } \\
, " \\
, " \\
, " \\
,\end{array}$ & $\begin{aligned} & \text { SCL }=\text { semi-confluent lysis } \\
& \mathbf{C L}=\text { confluent lysis } \\
&<\mathrm{SCL}\}=\text { intermediate } \\
&<\mathrm{CL}\} \text { degrees of lysis } \\
& \mathrm{OL}=\text { confluent "opaque" } \\
& \text { lysis (opacity due to } \\
& \text { heavy secondary } \\
& \text { growth) }\end{aligned}$ \\
\hline
\end{tabular}

Obviously, the amount of culture destroyed by a virulent phage is dependent on the size of the plaques; relatively few large plaques (often under $100)$ are required to produce confluent lysis, whereas many thousand minute plaques may be necessary to produce lysis of a similar degree. Each of the various adaptations of Vi-phage II has a characteristic plaque size, and when this is very small it may be difficult to use an R.T.D. that gives confluent lysis, because the phage concentration required would be so high that other types would be lysed. Under these circumstances a reading of semi-confluent lysis on the homologous type strain is accepted as adequate. Naturally, if a culture is lysed only by one of the pools of plaques, it is tested with the separate components of the pool in order to identify the phage to which it is sensitive. Examples of the characteristic appearances of the typing plates of a few Vi-phage types of $S$. typhi are shown in Fig. 2. (As these plates are intended to show the reactions of known types, the following spots are omitted: phage $\mathrm{A}(1,500), \mathrm{Vi}$-phages I, III, IV, V, VI, and the anti-O phage.)

Anomalous Reactions.-These can be divided into the following main groups:

(1) Cultures unlysed by any of the type adaptations of Vi-phage II : the absence of lysis may be due to the fact that the culture is basically resistant to Vi-phage II. It is, of course, impossible to prepare adapted typing phages for such cultures, but they may be sensitive to Vi-phages other than Vi-phage II. There are now five Vi-phages and an anti-O phage in routine use in the C.E.R.L. in addition to Vi-phage II, and it is sometimes possible to classify strains that are resistant to Vi-phage II by means of their characteristic reactions with these six unadapted phages. Nicolle, Pavlatou, and Diverneau (1954) have attempted a subdivision of such cultures by means of eight unadapted phages, only two of which are Vi-phages.

A culture may be resistant to the known typing adaptations of Vi-phage II because it is a new type for which no phage has yet been prepared. In this case it is often possible to prepare a typing phage for the new strain.

It is the present custom to designate all cultures that do not react with the typing adaptations of Viphage II as " untypable Vi strains," whether their lack of lysis is due to inherent resistance to $\mathrm{Vi}$ phage II or to the fact that they belong to new types. Fig. 3a shows the reactions of such a culture. In this illustration the spots of phage A $(1,500)$ and a pool of $\mathrm{Vi-phages} I$ and IV are included. The culture used, although resistant to the typing adaptations of Vi-phage II, is fully sensitive to the pool of Vi-phages I and IV.

(2) Cultures giving indeterminate reactions with the Vi-typing phages : many cultures are sensitive to a large number of the adapted typing preparations. The reactions may not exceed semi-confluent lysis with any of the phages, but may be confluent in some instances. Typhoid strains reacting to the Vi-typing phages in this way are known as "degraded Vi strains." A further discussion of this subject is given later. Fig. $3 \mathrm{~b}$ shows the reactions of such a culture. Here again, phage $A(1,500)$ is included in the test. Lysis by this phage is a useful indication of degradation in the cultures under examination. Because of the wide range of sensitivity of these strains to the typing phages, it is clearly impossible to assign them to specific types. On the other hand, such pattern reactions are usually constant for strains that are epidemiologically related to each other. Because of this, an identifiable pattern of phage susceptibility in strains of the typhoid bacillus is as useful to the epidemiological worker as is a specific type designation in tracing the source of infection.

It can be seen that when cross-reactions are sufficiently widespread and heavy the lytic pattern approaches that of type A (Fig. 2), which is 


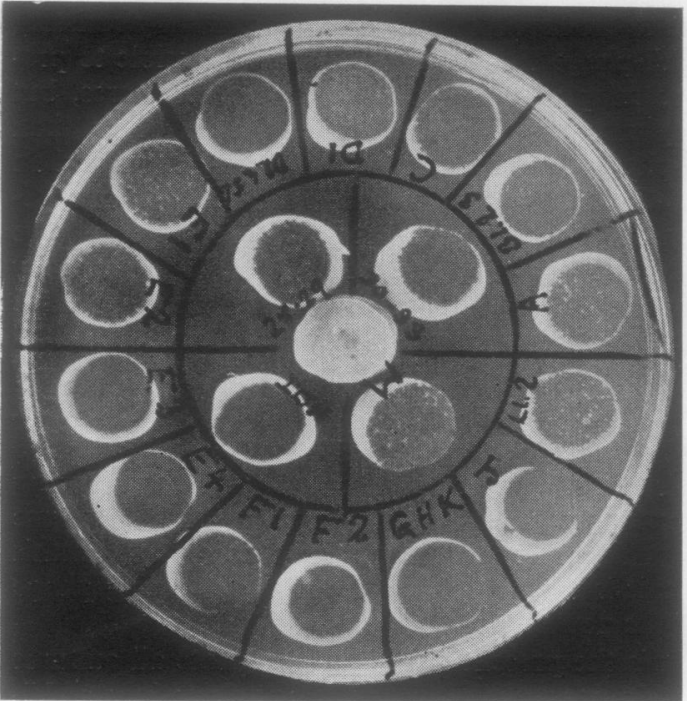

FIG. $2 a$.

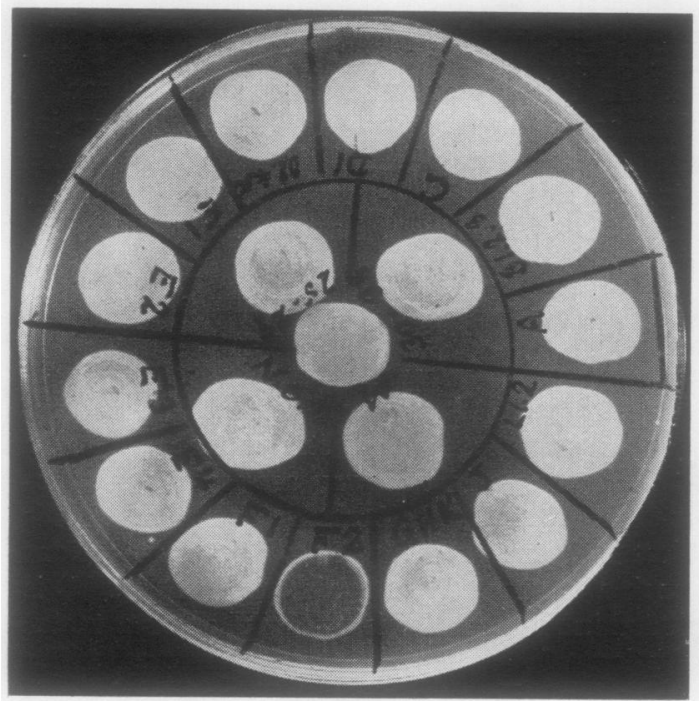

FIG. 20

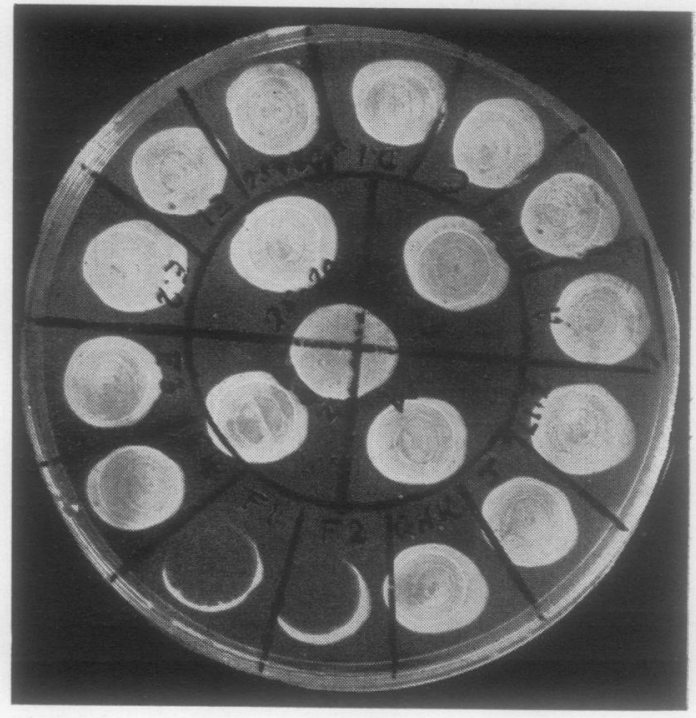

FIG. $2 b$,

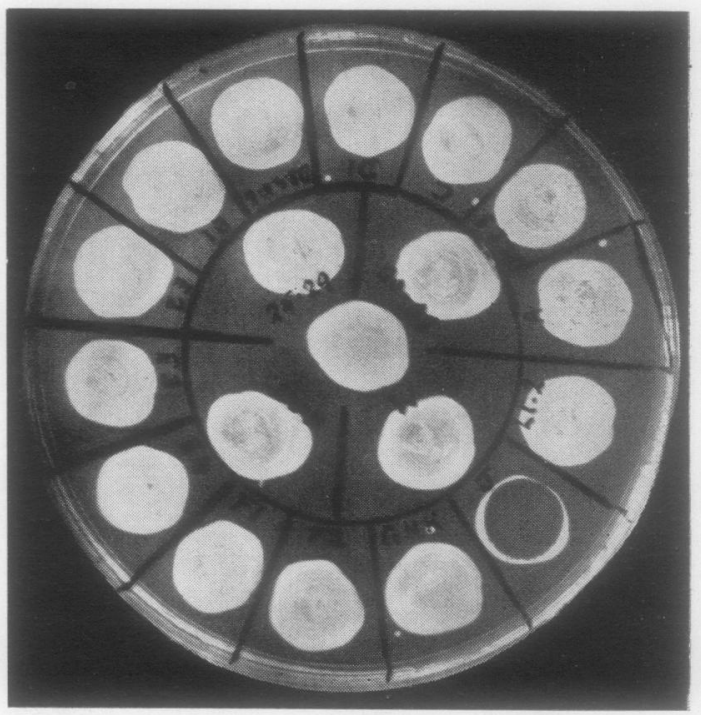

FIG. $2 d$.

FIG. 2. $-S$. typhi: reactions of Vi-phage types $\mathbf{A}, \mathrm{F}_{1}, \mathrm{~F}_{2}$, and $\mathbf{J}$ with the adapted typing phages in routine test dilution. (a) $\mathrm{Type} A$. (b) Type $F_{1}$. (c) Type $F_{2} . 【(d)$ Type J.

sensitive to all the Vi-typing phages. On occasion, the only difference between what might be termed a degraded strain and type $A$ is absence of lysis with one or two phages. Nicolle and his co-workers (see Nicolle, Hamon, and Edlinger, 1953) have suggested that such strains be designated as type $A$ with an indication of the gaps in the lytic pattern.

Some cultures of type $\mathbf{N}$ cross-react with the D group of phages. Such strains were first recog- nized some years ago by Craigie (see Felix, 1943) 0 and were given the specific type designation of $D_{3}$. This designation was abandoned, however, because $\stackrel{\mathcal{?}}{+}$ it was observed by Craigie (Craigie and Felix, $\square$ 1947) that incubation of the typing plates at temperatures higher than $38^{\circ} \mathrm{C}$. diminished the reac- $\stackrel{\mathbb{Q}}{\Omega}$ tion with phages of the $D$ group and the cultures $\stackrel{\mathbb{Q}}{\Omega}$ emerged as pure type $N$. Nicolle and his co- $\bar{O}$ workers have designated cultures giving this type of reaction as " $N+D_{1}$ " (Nicolle et al., 1953). 


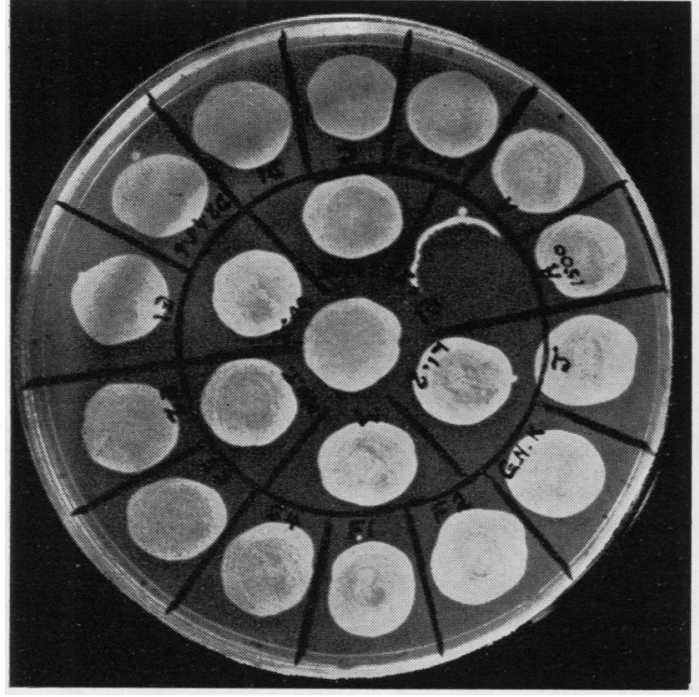

Fig. 3a.

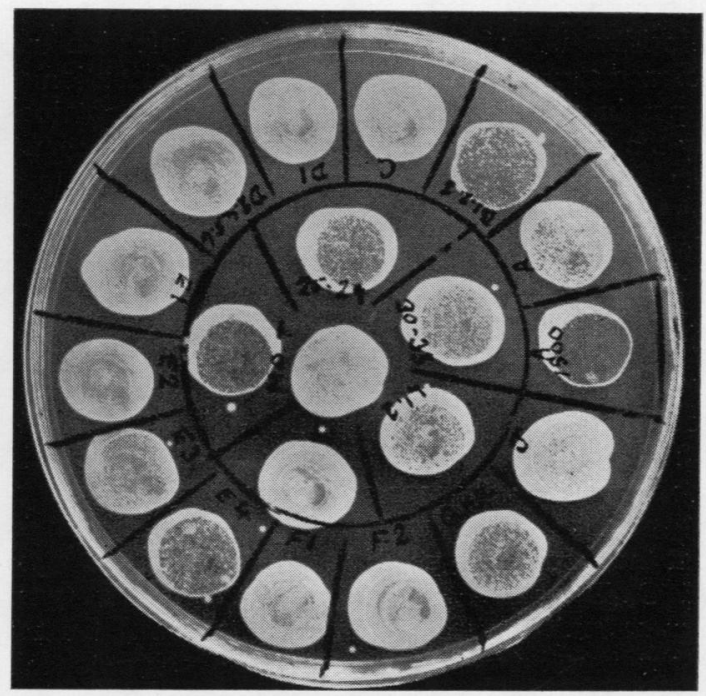

FIG. $3 b$.

FIG. 3.-S. typhi: (a) Untypable Vi strain. (b) Degraded Vi strain.

Any culture that does not give a clear-cut reaction is subjected to special investigation in an attempt to identify the type. These special tests are carried out because anomalous phage-typing results on the original culture may be due to the fact that it is undergoing variation and is producing clones of different degrees of type specificity.

It is, of course, obvious that if a culture does not possess the $\mathrm{Vi}$ antigen it cannot be typed with the adaptations of Vi-phage II. This has important implications for the persons who send cultures for enteric phage typing. As mutation of S. typhi from the Vi-positive to the Vi-negative state may take place quite rapidly in vitro, it is preferable to pick cultures intended for Vi-phage typing from primary platings on selective media such as deoxycholate-citrate or Wilson-Blair agar, and to prepare a pooled subculture of 12 colonies. Naturally, this is not always possible, as very few colonies of the typhoid bacillus may grow in primary platings. If the organism is isolated only after enrichment in fluid media, it is even more important to prepare a pool of at least a dozen colonies for phage typing. The selection of a single colony entails the risk that it may be a clone descended from a Vi-negative cell, in which case the culture cannot be phage typed. As the original plates may be discarded before it is discovered that a Vi-negative variant has been selected, it may be necessary to attempt to re-isolate the organism from the patient in order to obtain a Vi-typing result. If the strain cannot be isolated again, valuable epidemiological information is lost.
Frequency Distribution of the Vi-types of Salmonella typhi.-Table III shows the percentage distribution of the various Vi-types of $S$. typhi in England and Wales during 1954. This distribution was calculated on the basis of foci of infection and not by individual cultures. An outbreak of cases due to the same Vi-type known to be related to each other, or a number of apparently sporadic cases due to one Vi-type in a small area during a short period, are treated as a single event in Table III. Felix $(1950,1951,1955)$ drew attention to the need for analysing Vi-type incidence in this way. The true endemic frequency distribution of the

TABLE III

INCIDENCE OF SALM. TYPHI INFECTIONS DURING PERIOD JANUARY 1-DECEMBER 31, 1954 (Analysis by foci only)

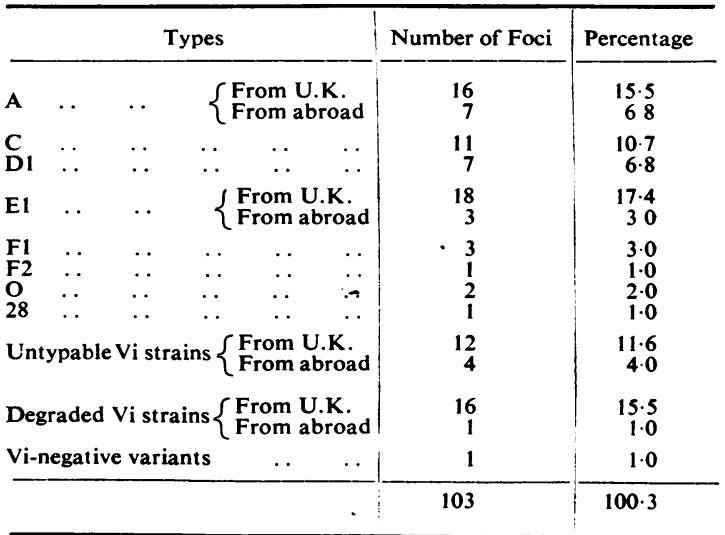


various types is more accurately arrived at by the consideration of possible sources of infection due to each type than by using the gross distribution of types in all cultures examined. Figures obtained by the latter method will be weighted by the occurrence of outbreaks due to one or a few types. Thus, what might be termed "gross type distribution"-arrived at by including all cultures in the analysis - tends to vary from year to year, whereas net distribution, which is the analysis by foci of infection, is remarkably constant. This is understandable when it is realized that the ultimate source of all cases of typhoid fever is the chronic carrier, and the distribution of chronic carriers in the population changes only very slowly.

The commonest type in this country, as throughout the world, is type E1 ; following this are types $A, C, D_{1}$, and $F_{1}$. The rarer indigenous types appear and disappear from year to year. On the other hand, types known to be foreign to this country are of exceptional occurrence, and, because the countries to which they are indigenous are known, it is usually possible to trace their origin. A very small number of persons in Great Britain are chronic carriers of non-indigenous types. These patients were infected abroad. A few outbreaks have been traced to such carriers, and, of course, the identification of the source of infection is greatly simplified by the fact that the types are rare. The position is much more difficult when an outbreak due to a common type such as type $A$ occurs, for about $15 \%$ of all sources of typhoid infection in Great Britain are type $A$ and a number of carriers of this type may inhabit a given area. The number of known chronic typhoid carriers in Great Britain is about 150 , but this is probably only a fraction of the total figure. In addition, there are many typhoid carriers in mental hospitals who are not included in this estimate. As typhoid bacilli from all carriers may infect the local sewage, mental hospital carriers cannot be excluded as possible sources of infection of the population at large.

It is now possible (see Felix, 1955) to survey the results of eight years of international cooperation in this field. As a result of the formation of the International Committee for Enteric Phage Typing through its national laboratories, now numbering 56 in 29 countries, much information has been gained concerning the distribution of the phage types of Salmonella typhi. It is clear, for example, that type $E_{1}$ is the commonest throughout the world. Following this are, successively, types $A, D_{1}$, and $C$. Although some types have a global distribution, others are characteristic of particular countries. If a type that is not indigenous to a given country is isolated from a case of typhoid fever, it is sometimes possible, knowing the countries in which the type is prevalent, to trace its origin. Thus the international exchange of information has a real value.

Examples of Reliability of Typhoid Vi-typing Method.-A few instances chosen from the routine work of the Central Enteric Reference Laboratory will serve to demonstrate the remarkable accuracy of the method of typing the typhoid bacillus with the adapted preparations of Vi-phage II.

In June, 1948, three children who drank water from the Wallington River contracted typhoid fever due to Vi-phage type $E_{1}$. During the investigations following these cases, typhoid bacilli of the same phage type were isolated from swabs (Moore, 1948, 1950) immersed at the places on the river visited by the children. The organism was traced up river to a tributary stream and thence to a sewage outflow, through the sewage system, and finally to a single house where a chronic carrier excreting phage type $E_{1}$ was found to be living. The investigation ended one year after the children had been infected and two and a half miles upstream from the point at which they had acquired the disease. It was controlled throughout by phage typing (see Lendon and Mackenzie, 1951). Typhoid bacilli belonging to various Vi-types are not uncommon in river water, and without the precision of phage typing this investigation would have been much more difficult to conclude satisfactorily.

Another example of the stability of phage types was found in a chronic carrier who was infected with Vitype $D_{6}$, which is not indigenous to Great Britain. It was discovered that the patient concerned had had typhoid fever some 20 years earlier in India, where type $D_{6}$ is relatively common.

Yet a further example is quoted in the Report of the Ministry of Health for 1953. A strain belonging to Vi-phage type $\mathrm{K}$ was isolated in 1942 from a woman who had been evacuated from Bromley, in Kent, to Somerset. Type $\mathrm{K}$ is rare in this country but is indigenous to India. In 1953 the same type was isolated from two patients at the Bromley Hospital, and it was $N$ found on investigation that these worked in a private $N$ hotel at which the first patient of the series had stayed $\omega$ in 1942. It was finally discovered that the wife of the owner of the hotel had had typhoid fever in India in 1935 and that she was excreting type $K$ in her faeces.

During the summer of 1955 typhoid cultures were $\stackrel{\circ}{+}$ received from six widely separated parts of England $\square$ within a period of eight days. All these cultures $\vec{O}$ were found to belong to Vi-type 34 , one of the $12 \stackrel{\vec{D}}{\stackrel{D}{*}}$ recently identified types mentioned earlier. Seven $\frac{\rho}{\mathbb{D}}$ patients were concerned in this outbreak. The in- $\varrho$ formation about the patients was incomplete at the time the earlier cultures were received in the C.E.R.L. The first two cultures arrived on consecutive days 
from Cardiff and London respectively, and the phagetyping results on each were available within 24 hours. Because type 34 is rare, it was assumed that the patients from whom the cultures had been isolated had probably been infected from a common source. Inquiries revealed that all the patients had visited the same hotel in a particular city in Spain within a given period, and it is believed that this was the source of their infection.

These examples could be multiplied many times, and numerous accounts have been published that demonstrate the value of the Vi-phage typing method (see, for example, Bradley, 1943 ; Felix, 1943, 1944; Warren and Goldie, 1945 ; Cruickshank, 1947 ; Lie Kian Joe, 1949 ; Smith, 1950 ; Dhayagude and Banker, 1951; Felix, 1951; de Blasi and Buogo, 1952; Nicolle et al., 1953; Brandis, 1955; Felix, 1955 ; Rische, 1954, 1955 ; Sampaio and Figueiredo, 1955a ; Eörsi, 1956). One of the results of the internationalization of the method has been that the identification of new types is soon followed by the distribution of the relevant type strains and typing phages to the various national laboratories. Thus, it is quickly possible to gain an idea of the incidence of the new types throughout the world. Because of the relatively small incidence of untypable strains, it can be assumed that all the common Vitypes have been identified, but, as a rare type may at any time become epidemiologically important, it is worth while for the time being to continue the identification of new types.

Of course, the method has its limitations. If one or a few types predominate in a particular country, or if a high proportion of strains cannot be typed, the value of the technique is restricted and other criteria must be sought for the subdivi- sion of the common types, or for distinguishing untypable strains from each other. Biochemical methods have been tried as an aid to Vi-phage typing when such limitations are encountered (see Olitzki, Shelubsky, and Strauss, 1948 ; Olitzki and Shelubsky, 1949 ; Felix and Anderson, 1951b ; de Blasi and Buogo, 1952 ; Pavlatou and Nicolle, 1953). Here again, however, bacteriophages offer the most promising possibilities, and Nicolle, Pavlatou, and Diverneau (1953) have been able to subdivide 600 type $\mathrm{A}$ strains into nine subtypes, and 200 untypable strains into seven subgroups by means of additional phages.

\section{Salmonella paratyphi B}

The phage typing of $S$. paratyphi B was introduced by Felix and Callow (1943, 1951). This scheme, which originally contained four phage types, now comprises 10 main types and subtypes. to which have been added a number of "variations." The latter show constant deviations from the major reaction patterns with the typing phages. The total number of distinguishable types, subtypes, and variations is at present over 30 . The paratyphoid B typing phages were at first thought to be adaptations of a single phase, analogous to the typhoid Vi-typing phages, but this was later found to be true only to a limited extent and several are distinct phages. Moreover, most of them are temperate phages, and there is little doubt that in a number of instances they arose as unsuspected contaminants from phages carried by the bacterial cultures used for propagating the phages it was hoped to adapt. Type identification by this method is not based on the specific reaction of a type of the organism with a single adapted phage

TABLE IV

REACTIONS OF TYPE STRAINS OF SALMONELLA PARATYPHI B WITH ROUTINE TEST DILUTIONS OF THE TYPING PHAGES

\begin{tabular}{|c|c|c|c|c|c|c|c|c|c|c|c|c|c|c|}
\hline \multirow{2}{*}{\multicolumn{4}{|c|}{ Type Strains }} & \multirow{3}{*}{$\frac{1}{C L}$} & \multirow{3}{*}{$\frac{2}{C L}$} & \multicolumn{7}{|c|}{ Typing Phages } & \multirow{3}{*}{$\frac{\text { Dundee }}{<\mathrm{SCL}}$} & \multirow{3}{*}{$\frac{1010}{-}$} \\
\hline & & & & & & \multirow{2}{*}{$\frac{3 a}{++}$} & \multirow{2}{*}{$\frac{3 \mathrm{aI}}{++}$} & \multirow{2}{*}{$\frac{3 b}{-}$} & \multirow{2}{*}{$\frac{\text { Jersey }}{\mathrm{CL}}$} & \multirow{2}{*}{$\frac{\text { Beccles }}{-}$} & \multirow{2}{*}{$\frac{\text { Taunton }}{-}$} & \multirow{2}{*}{$\frac{\text { B.A.O.R. }}{-}$} & & \\
\hline 1 & . & .. & . & & & & & & & & & & & \\
\hline 2 & . & . & . & - & $\overline{C L}$ & - & - & - & - & - & - & - & SCL & - \\
\hline $3 a$ & . & . & . & - & - & $<\mathrm{CL}$ & $<\mathrm{CL}$ & OL & - & $<\mathrm{CL}$ & $<\mathrm{CL}$ & OL & $<\mathrm{CL}$ & $\mathrm{CL}$ \\
\hline $3 \mathrm{aI}$ & . & .. & . & - & - & $<\mathbf{C L}$ & $<\mathrm{CL}$ & - & - & - & - & - & $<\mathrm{CL}$ & $\mathrm{CL}$ \\
\hline $3 b$ & .. & .. & . & - & - & - & - & $\mathrm{OL}$ & - & $<\mathrm{CL}$ & $<\mathrm{CL}$ & OL & $<\mathrm{CL}$ & $\mathrm{CL}$ \\
\hline Jerse & & . & . & - & - & - & - & - & $<\mathrm{CL}$ & $<\mathrm{CL}$ & $<\mathrm{CL}$ & $<\mathrm{CL}$ & $<\mathbf{C L}$ & $C L$ \\
\hline Becc & & .. & . & - & - & - & - & - & - & $\langle\mathbf{C L}$ & $<\mathrm{CL}$ & - & $<\mathrm{CL}$ & CL \\
\hline Taur & ton & . & . & - & - & - & - & - & - & - & $<\mathrm{CL}$ & - & $<\mathrm{CL}$ & $\mathrm{CL}$ \\
\hline B.O & A.R. & . & .. & - & - & - & - & - & - & - & - & $\mathrm{OL}$ & - & SCL \\
\hline Dun & dee & $\ldots$ & . & - & - & - & - & - & - & - & - & - & $<\mathrm{CL}$ & $C L$ \\
\hline
\end{tabular}


TABLE V

REACTIONS OF RECOGNIZED VARIATIONS OF PHAGE TYPES OF SALMONELLA PARATYPHI B WITH ROUTINE TEST DILUTIONS OF TYPING PHAGES

\begin{tabular}{|c|c|c|c|c|c|c|c|c|c|c|c|c|}
\hline \multirow{2}{*}{ Type } & \multirow{2}{*}{ Variation } & \multicolumn{7}{|c|}{ Typing Phages } & \multirow[b]{2}{*}{ Taunton } & \multirow[b]{2}{*}{ B.A.O.R. } & \multirow[b]{2}{*}{ Dundee } & \multirow[b]{2}{*}{1010} \\
\hline & & 1 & 2 & $3 a$ & $3 \mathrm{aI}$ & $3 b$ & Jersey & Beccles & & & & \\
\hline 1 & \begin{tabular}{ll}
\multicolumn{2}{l}{ Common } \\
Var. & 1 \\
$"$, & 2 \\
$"$, & 3 \\
$"$, & 4 \\
$"$, & 5
\end{tabular} & $\begin{array}{l}C L \\
C L \\
C L \\
C L \\
C L \\
C L\end{array}$ & $\begin{array}{l}C L \\
C L \\
C L \\
C L \\
C L \\
C L\end{array}$ & $\begin{array}{c}++ \\
+++ \\
\text { CL } \\
\text { CL } \\
\text { CL } \\
\text { CL }\end{array}$ & $\begin{array}{l}++ \\
+++ \\
\mathbf{C L} \\
\mathbf{C L} \\
\mathbf{C L} \\
\mathrm{CL}\end{array}$ & $\begin{array}{l}- \\
+\overline{S C L} \\
<\mathrm{CL} \\
<\underline{C}\end{array}$ & $\begin{array}{l}C L \\
C L \\
C L \\
C L \\
C L \\
C L\end{array}$ & $\begin{array}{l}\overline{S C L} \\
\text { SCL } \\
= \\
=\end{array}$ & $\begin{array}{c}\underset{\mathbf{S C L}}{<\mathbf{C L}} \\
= \\
=\end{array}$ & $\begin{array}{c}-\overline{+}+ \\
+\overline{O L} \\
\text { OL } \\
\text { OL } \\
\text { OL }\end{array}$ & $\begin{array}{l}\text { SCL } \\
\text { SCL } \\
<C L L \\
\text { SCL } \\
\text { SCL } \\
\text { SCL }\end{array}$ & $\begin{array}{l}\overline{C L} \\
C L \\
C L \\
\overline{C L}\end{array}$ \\
\hline 2 & $\begin{array}{l}\text { Common } \\
\text { Var. } 1\end{array}$ & $\overline{-}$ & $\begin{array}{l}C L \\
C L\end{array}$ & - & $=$ & - & $=$ & $\overline{S C L}$ & $\overline{\mathrm{SCL}}$ & 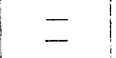 & $\begin{array}{l}\text { SCL } \\
\text { SCL }\end{array}$ & $\overline{C L}$ \\
\hline $3 \mathbf{a}$ & \begin{tabular}{ll}
\multicolumn{2}{l}{ Common } \\
Var. & 1 \\
., & 2 \\
$\because$. & 3 \\
$\because$. & 4 \\
$\because$. & 5 \\
., & 6 \\
\end{tabular} & $\begin{array}{l}= \\
= \\
= \\
=\end{array}$ & $\begin{array}{l}= \\
= \\
= \\
=\end{array}$ & $\begin{array}{l}<\mathbf{C L} \\
<\mathbf{C L} \\
<\mathbf{C L} \\
<\mathbf{C L} \\
\mathbf{C L} \\
\mathbf{C L} \\
<\mathbf{C L} \\
\end{array}$ & $\begin{array}{l}<\mathrm{CL} \\
<\mathrm{CL} \\
<\mathrm{CL} \\
<\mathrm{CL} \\
<\mathrm{CL} \\
\quad \mathbf{C L} \\
<\mathrm{CL}\end{array}$ & $\begin{array}{r}\text { OL } \\
\text { OL } \\
\text { OL } \\
\text { OL } \\
+++ \\
\text { SCL } \\
<\mathrm{OL} \\
\end{array}$ & $\begin{array}{l}\overline{-} \\
\overline{\mathrm{CL}} \\
\overline{-} \\
\overline{-}\end{array}$ & $\begin{array}{l}<\mathrm{CL} \\
<\mathrm{CL} \\
= \\
= \\
\overline{-} \\
<\overline{C L}\end{array}$ & $\begin{array}{l}<\mathrm{CL} \\
<\mathrm{CL} \\
\overline{-} \\
\overline{-} \\
\overline{\mathrm{C} L}\end{array}$ & $\begin{array}{c}\quad \overline{O L} \\
\overline{O L} \\
<\bar{C} L \\
<\overline{C L} \\
\bar{O} L\end{array}$ & $\begin{array}{l}<\mathbf{C L} \\
<\overline{C l} \mathbf{} \\
<\mathbf{C L} \\
<\mathbf{C L} \\
++++ \\
<\mathbf{C L}\end{array}$ & $\begin{array}{r}C L \\
C L \\
C L \\
C L \\
C L \\
<S C L \\
-\end{array}$ \\
\hline $3 \mathrm{al}$ & \begin{tabular}{ll}
\multicolumn{2}{l}{ Common } \\
Var. & $\mathbf{1}$ \\
, & 2 \\
, & 3 \\
$"$, & 4 \\
, & 5 \\
, & 6 \\
, & 7
\end{tabular} & $\begin{array}{l}= \\
= \\
= \\
=\end{array}$ & $\begin{array}{l}= \\
= \\
= \\
=\end{array}$ & $\begin{aligned}<C \mathbf{C L} \\
<\mathrm{CL} \\
<\mathrm{CL} \\
\mathbf{C L} \\
\mathbf{O L} \\
\mathbf{C L} \\
\mathrm{OL} \\
\mathrm{OL}\end{aligned}$ & $\begin{aligned}<C \mathbf{C L} \\
<\mathrm{CL} \\
<\mathrm{CL} \\
<\mathrm{CL} \\
\quad \mathrm{OL} \\
\quad \mathrm{CL} \\
\quad \mathrm{OL} \\
\quad \mathrm{OL}\end{aligned}$ & $\begin{array}{l}= \\
= \\
= \\
=\end{array}$ & $\begin{array}{l}= \\
= \\
= \\
\overline{+}+\end{array}$ & $\begin{array}{l}<\bar{C} L \\
<C L L \\
<\overline{C L} \\
\overline{-} \\
\overline{S C L}\end{array}$ & $\begin{array}{l}<\bar{C} L \\
<C L \\
<\overline{C L} \\
+\overline{+}+ \\
<\bar{C} L\end{array}$ & $\begin{array}{c}+\overline{+} \\
\overline{+} \\
+\overline{\mathbf{S C L}} \\
\overline{-} \\
++\end{array}$ & $\begin{array}{l}<\text { CLL } \\
<C L \\
<C L \\
\overline{C C L} \\
\mathbf{S C L} \\
+\overline{+}+\end{array}$ & $\begin{array}{c}<C L \\
C L \\
C L \\
C L \\
C L \\
C L \\
\text { SCL } \\
O L\end{array}$ \\
\hline $3 b$ & \begin{tabular}{ll}
\multicolumn{2}{l}{ Common } \\
Var. & 1 \\
,$\because$ & 2 \\
$\ddot{,}$ & 3 \\
$\ddot{y}$ & 4 \\
,, & 5
\end{tabular} & $\begin{array}{l}- \\
- \\
-\end{array}$ & $\begin{array}{l}= \\
= \\
=\end{array}$ & $\begin{array}{l}= \\
= \\
=\end{array}$ & $\begin{array}{l}= \\
= \\
= \\
\end{array}$ & $\begin{array}{l}\text { OL } \\
\text { OL } \\
\text { OL } \\
\text { OL } \\
\text { OL } \\
\text { OL }\end{array}$ & $\begin{array}{l}= \\
= \\
=\end{array}$ & $\begin{array}{l}<\mathrm{CL} \\
<\mathrm{CL} \\
= \\
\overline{\mathrm{SCL}}\end{array}$ & $\begin{array}{c}<\mathrm{CL} \\
<\underline{\mathbf{C L}} \\
\bar{Z} \\
<\underset{\mathrm{SCL}}{\mathrm{SCL}}\end{array}$ & $\begin{array}{r}\text { OL } \\
\text { OL } \\
<\overline{C L} \\
<\dot{S C L} \\
\end{array}$ & $\begin{array}{l}<\underline{C L} \\
<\overline{C L} \\
<\mathrm{CL} \\
<\text { SCL } \\
\text { SCL }\end{array}$ & $\begin{aligned} & C L \\
& C L \\
& C L \\
&<C L \\
& C L \\
& C L\end{aligned}$ \\
\hline Beccles & $\begin{array}{l}\text { Common } \\
\text { Var. } 1\end{array}$ & 二 & $=$ & 二 & - & - & 二 & $\begin{array}{l}<\mathrm{CL} \\
<\mathrm{CL} \\
\end{array}$ & $\begin{array}{l}<\mathrm{CL} \\
<\mathrm{CL}\end{array}$ & $<\overline{\mathrm{C}} \mathrm{L}$ & $\begin{array}{l}<\mathrm{CL} \\
<\mathrm{CL}\end{array}$ & $\begin{array}{r}\mathrm{CL} \\
<\mathrm{OL}\end{array}$ \\
\hline Dundee & $\begin{array}{l}\text { Common } \\
\text { Var. } 1\end{array}$ & - & $=$ & $=$ & $\overline{-}$ & - & $=$ & 二 & $\overline{-}$ & - & $\begin{array}{l}<\mathrm{CL} \\
<\mathrm{CL}\end{array}$ & $\mathrm{CL}$ \\
\hline
\end{tabular}

as it is in the case of S. typhi, but on the establishment of distinct reaction patterns of the various types with a number of phages. Table IV shows the reactions of the 10 main types and subtypes of $S$. paratyphi B with the typing phages at present in use.

Although there is a homologous phage that corresponds to each type, it is apparent that the characteristic patterns are of prime importance. Indeed, these patterns are so distinctive that deviations from them are epidemiologically significant and have served to characterize the variations of individual types and subtypes mentioned above. Thus, type $3 \mathrm{a}$ reacts with phages $3 \mathrm{a}, 3 \mathrm{aI}, 3 \mathrm{~b}$, Beccles, Taunton, B.A.O.R., and Dundee. A strain was identified in 1953, however, that was lysed by phages 3a, 3aI, 3b, and Dundee only. This was designated "type 3 a variation 2," and it has recently been shown that this reaction is epidemiologically stable (see Newell, 1955a). Table V, for which we are indebted to Miss B. R. Callow, shows most of the type variations of $S$. paratyphi B recognized at present.

Technique.-The technique of phage typing $S$. paratyphi B is identical with that of $S$. typhi. The organisms are grown in Difco broth to a turbidity of about $5 \times 10^{8}$ cells $/ \mathrm{ml}$. The Difco agar plates,

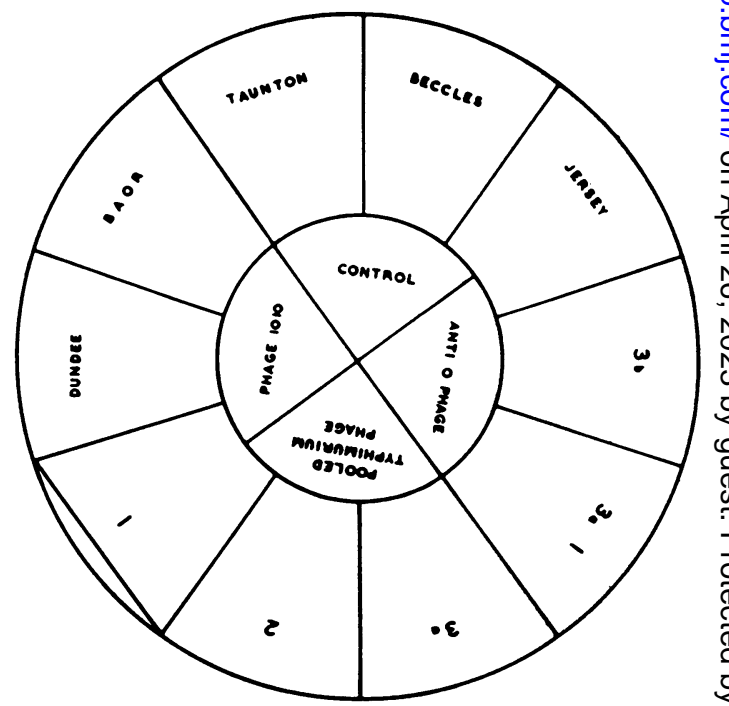

FIG. 4.-Method of marking plates for phage typing of $S$. paratyphi $B$. 


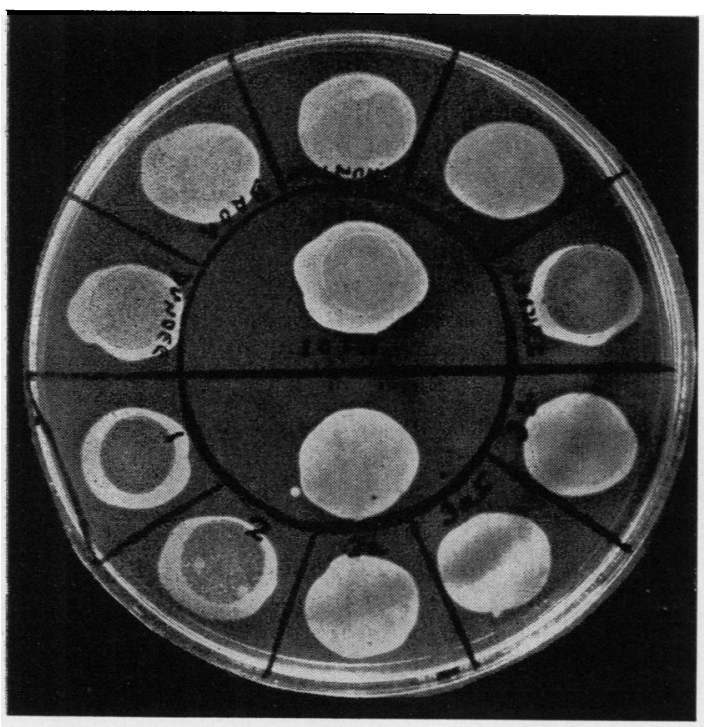

FIG. $5 a$.

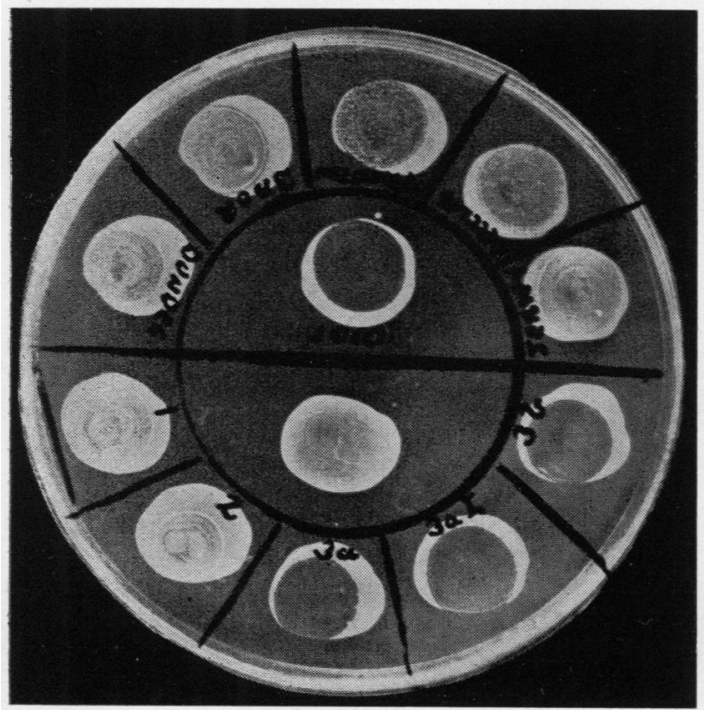

Fig. $5 c$.

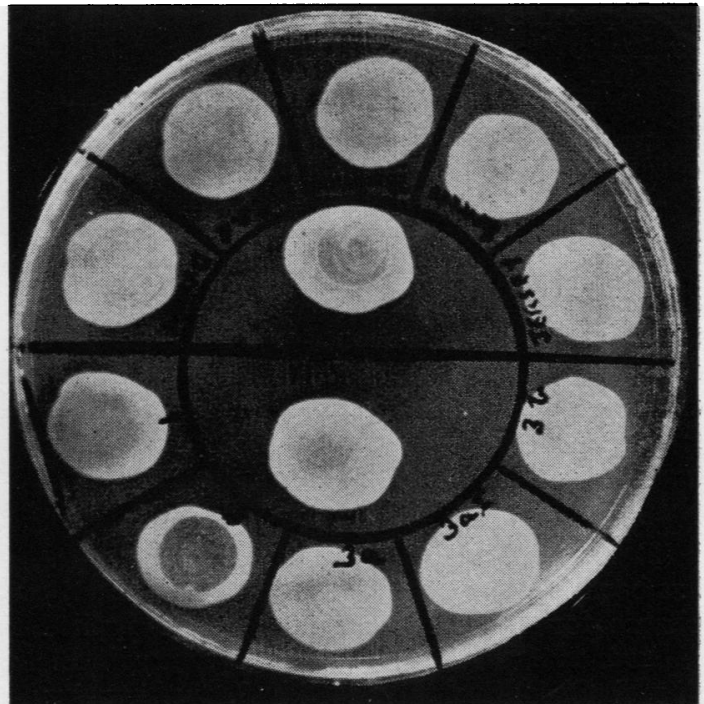

FIG. $5 b$.

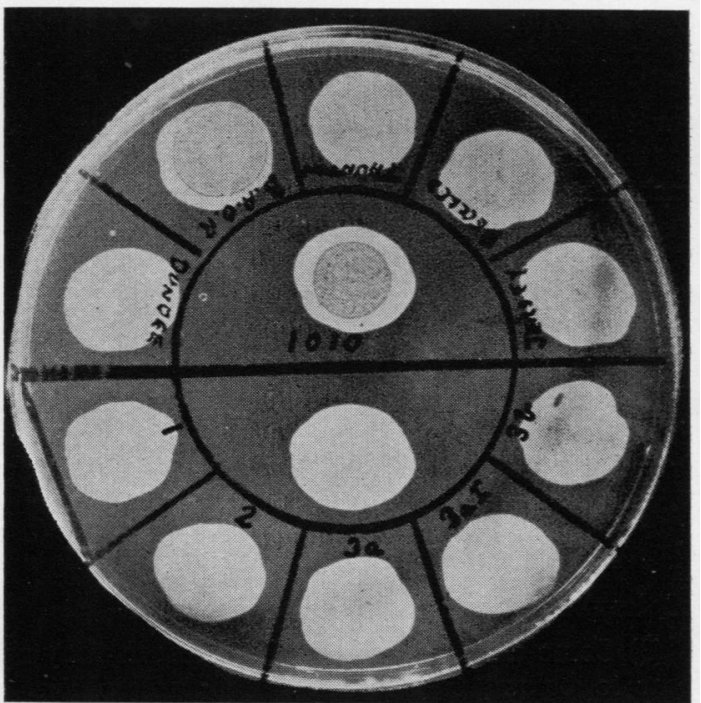

FIG. $5 d$.

FIG. 5.-Reactions of phage types 1, 2, 3a, and B.A.O.R. of $S$. paratyphi B with the typing phages in routine test dilution. (a) Type 1. (b) Type 2. (c) Type 3a. (d) Type B.A.O.R.

suitably dried, are marked as shown in Fig. 4 and the culture is spread in the various segments by means of the standard platinum loop.

The phages in R.T.D. are spotted on to the culture. A pool of $S$. typhimurium typing phages is always included in one segment, because cultures sent in as the paratyphoid B bacillus occasionally prove to be $S$. typhimurium. If no lysis occurs with the paratyphoid B typing phages, confluent lysis with the pool of $S$. typhimurium phages suggests that the strain under examination is $S$. typhimurium. First readings are taken with a $\times 10$ lens under direct and oblique lighting after five hours' incubation at $38.5^{\circ} \mathrm{C}$. and second readings after the completion of 24 hours' incubation.

Fig. 5 shows the reactions of some of the main types of $S$. paratyphi B.

Anomalous Reactions.-Reactions that do not conform to the pattern of any of the recognized 
paratyphoid $B$ phage types or type variations may be due to the following main causes.

(1) The culture may belong to a hitherto unidentified type of $S$. paratyphi B. In this case it may present a new reaction pattern with the typing phages sufficient for its identification, or it may be comp.etely resistant to the availabe phages. In the latter instance it is sometimes possible to obtain a phage that will lyse the new type, either from one of the existing preparations, or from a new source such as a lysogenic strain of $S$. paratyphi B, from another lysogenic salmonella, or from faeces or sewage filtrates (the last two sources are not recommended because of the non-specificity of the phages they may yield).

(2) The culture may not be $S$. paratyphi B. It has been indicated earlier that a pool of the typing phages for $S$. typhimurium is always applied to cultures sent to the C.E.R.L. as $S$. paratyphi B. As this gives confluent lysis with $S$. typhimurium it enables a number of cultures orginally thought to be $S$. paratyphi B to be correctly identified as $S$. typhimurium. However, cultures belonging to other salmonella serotypes may also be sent in error and these may or may not react with the paratyphoid $B$ typing phages, which are not specific for $S$. paratyphi B. When a lytic pattern is encountered that is not characteristic of one of the known phage types of $S$. paratyphi B the antigenic structure of the organism is always examined, and on a few occasions the cultures under scrutiny have proved to be other members of somatic Group B, or to belong to other somatic groups of the Kauffmann-White scheme. It may be mentioned in passing that $S$. abortus equi (somatic formula 4,12$), S$. newport $(6,8)$, and $S$. gallinarum, $S$. pullorum, and $S$. enteritidis $(9,12)$ are all sensitive to the paratyphoid $B$ typing phages although they do not show the type-specific patterns of lysis of the paratyphoid $B$ bacillus.

(3) Some cultures in a single outbreak may appear to belong to one pinage type and others to another. Of course, it is possible that more than one type may emanate from a single infecting source. This is not uncommon with infections due to heavy sewage contamination, and was recently shown to be probable in an outbreak due to Chinese frozen egg (Newell, Hobbs, and Wallace, 1955). However, double-type infections may be found when there is no reason to suspect that more than one type was originally concerned. In such instances it will usually be found that the lytic patterns of the two types present a partial similarity. An example of this occurred in June, 1955, during a paratyphoid outbreak due to phage type
Taunton in the south of England. One of the patients in this outbreak was found originally to be excreting phage type Dundee variation 1 . Reference to Tables IV and V will show that type Taunton differs from Dundee var. 1 in being sensitive to phages Taunton and 1010 (the latter phage does not define any single type but helps partly to define a number of types). Tests of single colonies picked from a culture isolated from this patient revealed that the strain consisted of a mixture of types Taunton and Dundee var. 1. A temperate phage was isolated from colonies showing the Dundee type of reaction, and it was found that lysogenization of the type Taunton colonies with it converted them into Dundee var. 1. Thus, it was assumed that the original type Taunton had probably been infected and lysogenized with a temperate phage in the intestine of the patient from whom the aberrantly reacting culture was isolated and that this lysogenization, by making the culture resistant to the typing phages Taunton and 1010 , had produced the type transformation. A similar incident involving two other phage types was detected within a few weeks of that described above, and again it was possible to show that a type-transforming phage was responsible for the difference between the two types, which were certainly epidemiologically uniform. The subject of type transformation will be discussed in more detail later. For the moment it is sufficient to say that, in spite of the fact that two examples of it were encountered within a few weeks of each other, it is evidently of exceedingly rare occurrence under natural conditions, because these occasions were the first in over 14 years' experience of paratyphoid B phage typing in the C.E.R.L.

Certain paratyphoid $B$ phage types present constant peculiarities in other characters. For example, type Jersey is always monophasic (Anderson, 1955b). This observation has been confirmed by the examination of a number of cultures of type Jersey isolated in different parts of Europe which were kindly sent to the C.E.R.L. by Dr. Pierre Nicolle, of Paris. Type Jersey is typical of the paratyphoid $B$ bacillus in most of its characters. It causes enteric fever, ferments dulcitol but not D-tartrate, and forms a slime wall. The question naturally arises whether the association between the monophasic character and the phage type of these strains is due to genetic linkage or chance. It has been possible to change the phage type of Jersey without altering its monophasic character. On the other hand, although it has been possible to change its flagellar antigen (Spicer, personal communication) without altering its phage type, it has not yet been made diphasic. 
All things considered, it seems probable that the association between the phage type and the monophasic character is accidental. If this is so, the interesting inference can be drawn that all strains of type Jersey are descended from a single variant in which the two characters happened to be associated by chance.

Practical Value of Phage Typing of Salmonella paratyphi B.-Many examples have been published of the value of the phage typing method for $S$. paratyphi $\mathrm{B}$, and the general rel ability of the scheme is of the same order as that of S. typhi (see King, 1944； Desranleau, 1947 ; Martin, 1947 ; Wallace and Mackenzie, 1947; Desranleau and Martin, 1950 ; Kennedy and Payne, 1950 ; Nicolle, Jude, and Buttiaux, 1950 ; Felix and Callow, 1951 ; Nicolle et al., 1953 ; Rische, 1954, 1955 ; Brandis, 1955 ; Felix, 1955; Sampaio and Figueiredo, 1955b). The technique has been internationally standardized in a manner similar to that used for typhoid phage typing, and it is now possible to compare the distribution of paratyphoid $B$ phage types in a number of countries.

Table VI shows the type distribution encountered in the United Kingdom during 1954. As with the figures for the Vi-phage types of $S$. typhi, the analysis is based on the incidence of foci of infection and not on the total number of cultures examined.

TABLE VI

INCIDENCE OF SALMONELLA PARATYPHI B INFECTIONS DURING THE PERIOD JANUARY 1-DECEMBER 31, 1954
(Analysis by foci only)

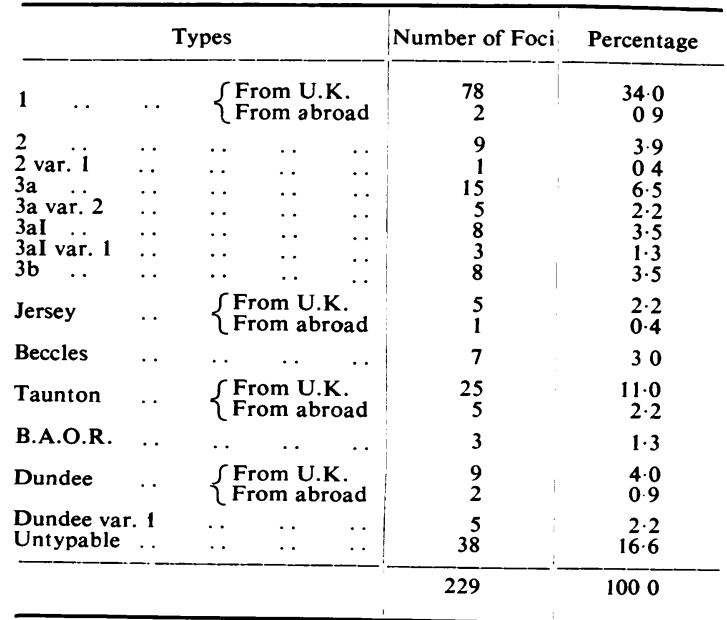

During the year 1954 the type distribution in order of frequency was as follows: types 1 , Taunton, 3a, Dundee, 2, 3aI and 3b, Beccles, 3a var. 2, Jersey and Dundee var. 1, B.A.O.R. and
2 var. 1. The proportion of untypable strains $(16.6 \%)$ is smaller than that found with the typhoid bacillus. In other European countries type Taunton is most frequently encountered. Desranleau and Martin (1950) have found that the commonest type in Canada is 3a. It is of considerable interest that the characteristic distribution of paratyphoid B phage types varies from continent to continent. However, there is at present no indication that special phage types are indigenous to some countries and not to others, as is found with certain typhoid Vi-phage types.

Striking results have been obtained in England from close collaboration between medical officers of health, epidemiologists, and laboratories. These results show that the prompt use of the information provided by the phage-typing method can identify the source of an outbreak of enteric fever with great precision. This was well exemplified by the tracing of outbreaks of paratyphoid B fever in Worthing and Weymouth to a single shipment of frozen whole egg imported from China (see Newell et al., 1955). Strong evidence was also obtained that a number of scattered cases of paratyphoid fever throughout this country were caused by a similar product, although in the latter instance the infecting phage type of $S$. paratyphi B (3a var. 2, which is distinct from 3a) was not isolated from the batches of egg concerned (Hobbs and Smith, 1955; Newell, 1955a, b ; Smith and Hobbs, 1955). The occurrence within a short interval of a number of scattered cases of paratyphoid B fever due to the same phage type is sufficient to indicate that some common factor is responsible for food contamination.

It has been stated that the enteric phage-typing systems are chietly of use where human carriers responsible for the dissemination of infection can be incriminated (Felix, 1955, 1956). That this is not so is shown by the major role played by phage typing in the demonstration of the spread of paratyphoid B fever by Chinese frozen egg. Although the human carrier is the ultimate source of infection with the typhoid and paratyphoid B bacilli, the routes by which the organism he excretes may reach the intestinal tract of his victims are many and varied. The great value of enteric phage typing lies in the fact that it enables us to relate the infecting organism in an outbreak to its source at whatever point in the infective chain this may be tapped: food, water, milk, sewage and, finally, under optimal conditions, the carrier.

It may be mentioned that for some years confectionery manufactured by commercial bakeries has probably been responsible for outbreaks of 
paratyphoid fever. Cockburn, Jameson, and Fenton (1951) and Thomson (1953) suggested that the paratyphoid B bacillus had gained access to the bakery in some product in frequent use. The epidemics described by Newell et al. (1955) were, however, the first in which definite proof of the source of contamination was obtained.

\section{Salmonella typhimurium}

Three methods have been used for phage typing this organism. The first was that of Felix and Callow (see Felix and Callow, 1943, 1951 ; Felix, 1956 ; Callow, to be published). This was developed on similar lines to the paratyphoid B typing scheme, and, like the latter, was originally thought to consist of a battery of phages all of which were adaptations of a single phage. It was later established, however, that most of the phages were distinct from each other, and there seems to be little doubt that many of them were derived from lysogenic strains on which attempts were being made to carry out specific phage adaptations. Fortunately, the heterogeneity of the typhimurium typing phages does not impair their value, and the reliability of the scheme is of the same high order as that of the typhoid and paratyphoid B bacilli. The fact that the chronic human carrier plays a negligible part in the epidemiology of typhimurium infections does not in any way limit the value of the typing scheme. Its use has made it possible to connect human outbreaks of food poisoning due to $S$. typhimurium with food preparations responsible for the infection, and with infected animals such as pigs, ducks, and poultry. Miss B. R. Callow, of the C.E.R.L., who played an important part in the development of the original scheme for typing $S$. typhimurium, is revising, improving and standardizing the existing typing scheme for this organism; the results of this work will be published elsewhere. For the moment, it is sufficient to mention only that the method in current use comprises 12 types and subtypes, with many additional variations that have been shown to be epidemiologically valid. The major phage types and subtypes of this organism are shown in Table VII.

The technique of setting up the tests and the methods of reading and interpretation are similar to those used for S. paratyphi B.

As with $S$. paratyphi $\mathrm{B}$, an instance has been found of an association between a particular phage type of $S$. typhimurium and a peculiarity of the organism, in this case, anaerogenicity. Anaerogenic cultures of $S$. typhimurium are rare; nevertheless, such strains have been isolated in this country intermittently since 1943 . They have sometimes been encountered in sporadic cases but have also been found in small outbreaks, and it has occasionally been possible to trace the outbreaks to infected turkeys (Pereira and Blaxland, 1955). Without exception all these anaerogenic strains have belonged to a type we designate 1a variation 1. In spite of the invariable association of this phage type with a metabolic peculiarity, no evidence is at present available to indicate that the association is due to anything but chance, which suggests, as in the case of the monophasic phage type Jersey of $S$. paratyphi B, that all the strains concerned originated from a single strain in which the characters happened to coincide fortuitously.

The second phage typing scheme used for S. typhimurium is that of Lilleengen (1948). This uses 12 phages of diverse origin and comprises 24 types. Lilleengen's system is in current use in Sweden. It is based on principles similar to the

TABLE VII

REACTIONS OF TYPE STRAINS OF SALMONELLA TYPHIMURIUM WITH ROUTINE TEST DILUTIONS OF THE TYPING PHAGES

\begin{tabular}{|c|c|c|c|c|c|c|c|c|c|c|c|c|}
\hline \multicolumn{2}{|c|}{ Test Strains } & \multicolumn{3}{|c|}{$\mathbf{I}$} & \multicolumn{5}{|c|}{ II } & \multicolumn{2}{|c|}{ III } & \multirow{2}{*}{$\frac{\text { IV }}{4}$} \\
\hline Group & Type & 1 & la & $1 \mathrm{~b}$ & 2 & $2 a$ & $2 b$ & $2 c$ & $2 d$ & 3 & $3 a$ & \\
\hline I & $\begin{array}{l}1 \\
1 \mathrm{va} \\
1 \mathrm{~b}\end{array}$ & $\begin{array}{l}\text { OL } \\
=\end{array}$ & $\begin{array}{l}\text { OL } \\
\text { OL } \\
\text { OL } \\
-\end{array}$ & $\begin{array}{l}<\mathbf{C L} \\
<\mathbf{C L} \\
<\mathbf{C L} \\
<\mathbf{C L}\end{array}$ & $\begin{array}{l}C L \\
C L \\
\overline{C L}\end{array}$ & $\begin{array}{c}\overline{C L} \\
\overline{C L}\end{array}$ & $\begin{array}{c}<\text { CL } \\
\text { OL } \\
\text { OL } \\
\text { OL }\end{array}$ & $\begin{array}{l}<\frac{\mathrm{CL}}{\mathrm{CL}} \\
<\overline{\mathrm{CL}}\end{array}$ & $\begin{array}{l}\text { OL } \\
\text { OL } \\
\text { OL } \\
\text { OL } \\
\end{array}$ & $\begin{array}{r}\text { OL } \\
\text { OL } \\
\text { OL } \\
<\mathbf{C L} \\
\end{array}$ & $\begin{array}{r}\text { OL } \\
\mathbf{O L} \\
<\mathbf{C L} \\
<\mathbf{C L} \\
\end{array}$ & $\begin{array}{l}\bar{z} \\
\bar{z}\end{array}$ \\
\hline II & $\begin{array}{l}2 \mathrm{c} \\
2 \mathrm{a} \\
2 \mathrm{~b} \\
2 \mathrm{c} \\
2 \mathrm{~d} \\
\end{array}$ & $\begin{array}{l}\bar{z} \\
\bar{z}\end{array}$ & $\begin{array}{l}= \\
\bar{z}\end{array}$ & $\begin{array}{l}= \\
\bar{z}\end{array}$ & $\frac{\mathrm{CL}}{\overline{\mathrm{CL}}}$ & $\begin{array}{r}\mathrm{CL} \\
\mathbf{C L} \\
<\overline{C L} \\
\end{array}$ & $\begin{array}{l}<\overline{\mathrm{CL}} \\
\mathbf{\mathrm { OL }} \\
<\overline{\mathrm{CL}} \\
-\end{array}$ & $\begin{array}{r}\mathbf{C L} \\
\mathbf{C L} \\
<\overline{C L} \\
\end{array}$ & $\begin{aligned} & <\overline{\mathrm{CL}} \\
\mathbf{O L} & <\overline{C L} \\
< & <\mathbf{C L}\end{aligned}$ & $\begin{array}{l}= \\
= \\
\end{array}$ & $\begin{array}{l}= \\
\bar{z}\end{array}$ & $\begin{array}{l}\bar{z} \\
\bar{z}\end{array}$ \\
\hline III & \begin{tabular}{|l}
3 \\
$3 a$ \\
\end{tabular} & $=$ & $=$ & + \pm & 二 & 二 & $+\overline{+}+$ & $=$ & $S \overline{C L}$ & $\begin{array}{c}\text { OL } \\
+ \\
\end{array}$ & $<\dot{\mathrm{C}}_{\mathbf{\mathrm { L }}}^{+}$ & $=$ \\
\hline IV & 4 & - & - & - & - & - & - & - & - & - & - & $<\mathrm{CL}$ \\
\hline
\end{tabular}

* Type 1a var. 1 was formerly designated type $3 b$.

This variation is always anaerogenic. 
scheme of Felix and Callow, but type distinction by Lilleengen's method often depends on differences in reaction pattern that would be considered by the English workers too small to be dependable. However, experience in Sweden seems to have shown Lilleengen's phage typing system for $S$. typhimurium to be a valuable epidemiological guide in outbreaks of food poisoning due to this organism.

A third method of phage typing S. typhimurium was suggested by Boyd (1950) and Boyd, Parker, and Mair (1951). This consists in the identification of types (designated "marks" by Boyd, Parker, and Mair) by the isolation and characterization of the phages they carry. It is probable that all strains of $S$. typhimurium are lysogenic, and, provided that the identification of the temperate phages can be carried out with sufficient accuracy, this method should be reliable, but it has the disadvantage of being more time-consuming than those described earlier.

\section{Other Salmonellas}

Phage-typing schemes for salmonellas other than those described have been devised for Salmonella paratyphi A (Banker, 1955); S. typhimurium (Lilleengen, 1948); S. dublin (Lilleengen, 1950 ; Smith, 1951c); S. enteritidis (Lilleengen, $1950) ; S$. gallinarum and $S$. pullorum (Lilleengen, 1952); and $S$. thompson (Smith, 1951a and b). Obviously, there is little point in producing a phage-typing method for an organism that is uncommon and therefore epidemiologically unimportant. Most of the common salmonellas are covered by the schemes just mentioned, and, while there may be room for improvement in individual schemes, there are few further serotypes to which it would be worth while applying the method. In Europe, however, it would probably be an advantage to have a typing scheme for $S$. newport. On the American continent $S$. montevideo and $S$. oranienburg are relatively common and are important contaminants of spray-dried egg (see M.R.C. Special Report, 1947 ; Edwards, Bruner, and Moran, 1948). They have caused a number of outbreaks of food poisoning and might repay attempts at subdivision into phage types.

\section{PREPARATION OF CULTURES FOR DESPATCH TO THE C.E.R.L. FOR ROUTINE PHAGE \\ TYPING}

The desirability of sending a representative sample of cultures has already been emphasized, and it has been pointed out that a pool of at least 12 colonies from primary platings is most satisfactory.
Cultures are frequently despatched by post in $20 \mathrm{ml}$. universal containers on rather moist agar slopes. By the time these arrive in the reference laboratory the agar has disintegrated and the culture consists of an agar pulp with a considerable amount of free fluid containing a high concentration of bacteria in suspension. The containers often develop a positive pressure and removal of the screw cap results in the release of an aerosol of the pathogenic organism-S. typhi, S. paratyphi B, or $S$. typhimurium. Moist Dorset egg slopes are also offenders in this respect. Special precautions must be taken to deal with these cultures, as their handling entails considerable risk.

Dorset egg medium is employed as a routine in the C.E.R.L. for storing and despatching cultures. The slopes are prepared in the usual way, in 3 in. $\times \frac{1}{2}$ in. unlipped tubes which are plugged with cotton wool. The tubes are then inverted and left at room temperature for three days, during which free moisture drains into the plugs. At the end of this time the plugs are replaced by sterile corks which have been immersed in a molten mixture of equal parts of paraffin wax (melting point $56^{\circ}$ C.) and vaseline. These tubes are suitable for the despatch of pathogenic bacteria by post, because they are robust and breakages in transit are rare. The dry surface of the medium and the lack of free fluid ensure that the culture is confined to the area of inoculation medium. However, universal containers or $5 \mathrm{ml}$. screw-capped bottles are equally satisfactory, provided that similar precautions are taken to eliminate free moisture.

\section{THE USE OF INFORMATION PROVIDED BY ENTERIC PHAGE TYPING}

It is obvious that cultures isolated from a single outbreak will belong to the same phage type, and, when the carrier responsible for the outbreak can be located, phage typing will indicate the identity of the organism he is excreting with the epidemic strain. Similarly, the investigation of outbreaks of enteric fever, or of food poisoning due to $S$. paratyphi B or S. typhimurium, can often be helped by the phage-typing method. There are special instances, however, in which phage typing offers proof of the epidemiological uniformity of strains that would otherwise be difficult to connect with each other. One of these is the occurrence of apparently sporadic cases due to the same phage type at intervals over a considerable period, either in the same area or in different areas. A striking example of this was published by Bradley (1943). Twenty-three cases of typhoid fever due to $\mathrm{Vi}$ phage type $D_{4}$ occurred over an area covering four 
counties during a period of two years. The infection was milk-borne and the source was eventually traced to a chronic carrier on a farm in Wiltshire 100 miles from some of the cases. Without the Vi-typing method the possibility of solving the problem presented by these scattered cases would have been very slender.

The second type of incident in which phage typing is specially valuable has similarities to that just described, and concerns the occurrence of the same phage type in widely different areas simultaneously. This suggests the existence of a single source of infection in the form of a foodstuff or food ingredient that is distributed on a national scale. The detection of persons infected with enteric pathogens is delayed initially by the rather long incubation period of the disease, and possibly still further, in the early cases of an outbreak, by late diagnosis. It is evident, therefore, that unless rapid action is taken once the diagnosis is established, the original source of infection may be difficult to trace. One of the great advantages of the existence of a central laboratory to which cultures are sent from all parts of the United Kingdom is that the simultaneous occurrence of a single phage type in different areas can be detected very quickly and the necessary epidemiological investigation made.

\section{THEORETICAL ASPECTS OF ENTERIC PHAGE TYPING}

\section{Salmonella typhi}

All adapted preparations of Vi-phage II are neutralized by an antiserum prepared by inoculating rabbits with phage $A$, and there is no doubt that all are modifications of the one phage. The discovery of a single phage with a remarkable lability of host range naturally aroused a good deal of curiosity concerning the nature of the process of adaptation of the phage, and that of the difference between the types of the typhoid bacillus it was able to distinguish.

Adaptation of Vi-phage II to the Vi-types of S. typhi.-Craigie and Yen (1938a) postulated that the evolution of the specific adaptations of Vi-phage II consisted in a process of selection of spontaneously occurring host-range mutants. These authors believed that the specificity of a given adaptation depended on which mutant had been selectively propagated on the Vi-type of $S$. typhi which it was able to lyse. Felix (1949) rejected this hypothesis on the grounds that it was unlikely that two such widely different organisms as the typhoid bacillus and Vi-phage II could evolve a considerable number of complementary mutants. However, it has recently been shown (Anderson and Felix, 1952, 1953a, c ; Anderson, 1955a ; Anderson and Fraser; 1955, 1956) that the process of selection of host-range mutants does, in fact, play a considerable part in the production of the typing adaptations of $\mathrm{Vi}$ phage II, but that an additional phenomenon is concerned in the adaptation of this phage. This is the modification of the host range of the phage by contact with the cells to which it is to be adapted. Changes of this nature were first described in phage by Luria and Human (1952) Bertani and Weigle (1953) and Weigle and Bertani (1953) observed similar changes in phage P 2 isolated from the lysogen:c strain of Escherichia coli of Lisbonne and Carrère $(1922,1923)$ and in phage $\lambda$ of lysogenic $E$. coli K-12. Many of the adaptations of Vi-phage II are due to changes of a similar nature to those observed in phages $P 2$ and $\lambda$ by Bertani and Weigle. A full description of this subject cannot be given here, but it may be stated briefly that "host-induced" modification (Luria and Human, 1952) in phage or, as we have preferred to call it, "phenotypic modification," is non-mutational in origin. A small proportion of particles of the phage undergo modification in host range only on contact with the host to which the phage can be adapted. The phage descended from these particles has acquired a high specificity for the new host and may have lost its capacity for attacking the strain for which it was formerly specific. Propagation of the new phage on the strain for which it was specific before the most recent adaptation reverses the change in every particle, so that the original host specificity is regained. Strains usually exist which the phage is able to attack to full titre, whatever adaptations it may have undergone. The following example will summarize the process.

Phage E1, which is the adaptation of Vi-phage II to type E1 of $S$. typhi, exhibits on type C about 1 in 1,000 of its titre on type E1. This is known as the efficiency of plating of phage E1 on type $\mathrm{C}$ and is usually expressed as $10^{-3}$. If single plaques are picked at random from such a titration on type $\mathrm{C}$ it will be found that the phage they contain has an efficiency of plating on type $\mathrm{E} 1$ as compared with type $\mathrm{C}$ of about $10^{-3}$. In other words, the phage has undergone a reversal of host range : its high specificity for type E1 has changed to a high specificity for type C. The plaques formed by phage $\mathrm{E} 1$ on type $\mathrm{C}$, and by phage $C$ on type E1, might be descended from a modification of the host range of randomly distributed particles of each phage by contact with cells of the respective types (i.e., a phenotypic change in the phage), or might 
originate from host-range mutants of the phage that have been selected by the types concerned. In the former case the change of host range of the phage requires the presence of cells of the respective $\mathrm{Vi}$ type of the typhoid bacillus; in the latter it is mutational and independent of the presence of the bacterium. The distinction between the two processes can be drawn by propagating the phages in cells of type A which, as Table I shows, is fully sensitive to all adaptations of Vi-phage II. Phages that have undergone a phenotypic change only are all converted to phage $\mathbf{A}$ when grown on type $\mathbf{A}$, and this conversion affects every phage particle. Thus, when phage $\mathrm{El}$ is grown on type $\mathrm{A}$ it is completely converted into phage $A$. Because of the totality of this change it is clear that phage $\mathrm{El}$ cannot be a mutant of Vi-phage II. Phage $C$ shows the same property. On the other hand, phage $D_{1}$ is propagated unchanged on type $A$ and is a host-range mutant of Vi-phage II. Fluctuation tests of the type originally devised by Luria and Delbrück (1943) show that the phenotypically changeable particles and host-range mutants of Vi-phage II have characteristic statistical distributions (Anderson and Fraser, 1956).

It is now believed that phage A is the "wild" type of Vi-phage II (Anderson and Fraser, 1955). Some of the Vi-typing adaptations of Vi-phage II in current use are phenotypic modifications of the phage; others are host-range mutants; and yet others are combinations of both types of change.

The Vi-type Specificity of S. typhi.-Vi-type specificity seems to be a characteristic of the typhoid bacillus that is independent of other features of the organism. Thus, fermentative properties show the same variability between different strains of the same type that they do between strains of different types, and no evidence exists to show that any Vi-type is constantly more virulent than others (Felix and Anderson, 1951b). The one character that is indissolubly linked to the $\mathrm{Vi}$ typing scheme is the possession of the $\mathrm{Vi}$ antigen. As this is necessary for the adsorption of the typing phages to the host cells, it is indispensable to the typing method. The amount of $\mathrm{Vi}$ antigen produced by the cells does not seem to affect their susceptibility to the typing phages. If a strain of the typhoid bacillus belonging to any Vi-type contains only a minority of organisms in the Vi-positive form, however, a large proportion of organisms will not be attacked by the phage, and lysis will be somewhat obscured by the heavy background growth produced by the resistant Vi-negative cells.

All Vi-types of $S$. typhi will absorb all the typing preparations of Vi-phage II, irrespective of their specificity (Craigie, 1940). The initial step in the process of lysis by the typing phages, that of adsorption, is thus independent of type specificity. This adsorption is lethal to the bacterial cells (Anderson and Fraser, 1956), but phage multiplication does not occur unless the type of the organism and the specificity of the phage are complementary.

It may be concluded, therefore, that type specificity of the organism is dependent on a different component of the Vi-antigen complex from that concerned with the adsorption of Vi-phage II, or that it resides in some other site in the soma of the typhoid bacillus. Craigie $(1942,1946)$ observed that many strains of $S$. typhi carried temperate phages which he called "latent" or "gamma" phages. He believed that carriage of these phages made their host strains resistant to the Vi-typing phages and called such strains " imperfect Vi forms." He lysogenized type A with a temperate phage isolated from type $D_{1}$ and found that the newly lysogenized culture reacted as type $D_{1}$. The significance of these observations does not seem to have been realized, however, and the subject was not pursued. It has been shown recently (Anderson, 1951; Felix and Anderson, 1951a; Anderson and Felix, 1953b) that type specificity in $S$. typhi is partly controlled by the carriage of temperate phages which have been called "type-determining" phages. These phages erect specific barriers to the multiplication of Viphage II that can be overcome only by particu.ar host-range mutants, or by closely related mutants, of the Vi phage (Anderson and Fraser, 1955). Seven distinct determining phages are now recognized. They have been given small-letter designations when the type carrying them is designated by a capital letter; and a number with a superscript prime sign when the type carrying them has a numerical symbol. The determining phages recognized at present are : $d_{1}, d_{6} f_{2}, t, 25^{\prime}, 26^{\prime}, 30^{\prime}$. Phages $f_{2}$ and $30^{\prime}$ have indistinguishable typedetermining properties and for present purposes can be considered as identical.

Naturally occurring Vi-types can be replicated in the laboratory by lysogenizing certain nonlysogenic types with suitable type-determining phages (Anderson, 1951; Felix and Anderson, 1951a ; Anderson and Felix, 1953b). This enables us to consider the lysogenically determined types on a structural basis (Anderson, 1955; Anderson and Fraser, 1955). Using the current convention of designating a lysogenic strain by the symbol of its non-lysogenic precursor followed by that of the carried phage in parenthesis, a series of formulae have been evolved for the lysogenically determined types. Thus, type D6, which consists of type $A$ carrying phage $d_{6}$, is given the symbol 
$A\left(d_{6}\right)$. Similarly, type $F_{2}$ is structurally $F_{1}\left(f_{2}\right)$, type 33 is $C\left(d_{6}\right)$, type 25 is $A\left(25^{\prime}\right)$; and so on.

The determining phages are serologically unrelated to Vi-phage II. They are not specific for the $\mathrm{Vi}$-positive form of the typhoid bacillus but will lyse the Vi-negative form and some will even attack other salmonellas. Moreover, they affect the sensitivity of the typhoid bacillus, not only to the Vi-typing phages and other Vi-phages, but also to entirely distinct non-Vi phages. This indicates that Vi-type specificity is not resident in the $\mathrm{Vi}$ antigen but is determined elsewhere in the soma of the cell.

The type specificity of the lysogenically determined Vi-types of the typhoid bacillus depends on two factors: the nature of the non-lysogenic precursor of the type, and that of the type-determining phage. The former changes the phenotype of Vi-phage II ; the latter is responsible for the selection of specific host-range mutants of the Vi-phage. The host range of the typing phages for such types thus presents a summation of the two changes, and if the "structural formula" of a lysogenically determined type is known, that is, its nonlysogenic precursor and the determining phage it carries, this host range can be predicted. There are several types that have not so far yielded determining phages but can only be lysed by host-range mutants of Vi-phage II. It is not yet known what controls this property of mutant selection, but it seems probable that the same type of barrier to multiplication of the "wild" type of Vi-phage II can be a constitutive part of the bacterial cell or can be erected by the presence of a prophage.

Loss of Type-specificity and Degradation in S. typhi.-It has been pointed out earlier that some cultures give widespread reactions with the $\mathrm{Vi}$ typing phages and that such cultures are reported as " degraded Vi strains." In reality, the designation covers cross-reactions that may arise from a number of causes. Some Vi-types normally have only a limited amount of resistance to heterologous adaptations of Vi-phage II in R.T.D. Such types are: $B_{1}, B_{2}, B_{3}, C, E_{1}, N, O, T$, and 29. The reactions given by these types must be regarded as expressions of the maximum specificity that the types are capable of attaining, and, as their reactions with the homologous typing phages are full and clear-cut, the cross-reactions are accepted as part of the normal characteristics of the types. The sensitivity of some types to particular heterologous typing phages is as full as that to their homologous typing preparations. Some of these cross-reactions are due to the possession by certain Vi-types of similar determining phages or similar non-lysogenic precursors (Anderson, 1955a; Anderson and Fraser, 1955).

On the other hand, the change termed degradation may manifest itself in cultures belonging to specific Vi-types. It may occur uniformly throughout a culture, so that the examination of singlecolony lines will yield sensibly the same typing picture as that of the parent strain. Or it may affect only certain cells, in which case selection of single colonies will show that a proportion of cells have retained their full specificity. The reaction of the whole culture to the Vi-typing phages is then the mean of the specific and degraded elements in it. In this case the degradation process clearly occurs in a stepwise fashion in the culture and it may thus be different in origin from the uniform degradation referred to above. In the limit, degradation renders strains fully sensitive to all the Vi-typing phages; in other words, the cultures react as type A. Again, this change may occur in a single step, in which case single-colony selection will reveal that the culture consists of a mixture of the specific type and type A. Alternatively, all stages of degradation may be found in a strain, the specific type lying at one end of the process, and type $A$ at the other, with progeny of varying degrees of lowered type specificity occupying intermediate positions.

One case in which degradation has a clearly definable cause is that of the loss of determining phages by lysogenic Vi-types, although the justification of the use of the term degradation is doubtful in this context and "dissociation" seems more suitable. It was shown (Anderson, 1951) that type $F_{2}$, which reacts with phage $F_{2}$ only (see Table I), may lose the determining phage $\mathrm{f}_{2}$; if this occurs, it becomes type $F_{1}$ which is sensitive to phages $F_{1}$ and $F_{2}$. $A$ number of other instances of this nature may be quoted, in each of which the diminution or complete loss of type specificity has a clearly identifiable cause-loss of a determining phage. On the other hand, loss of specificity in a lysogenically determined Vi-type may be due to changes in the non-lysogenic moiety of the complex. An example of this change in type $D_{4}$ was quoted by Anderson and Felix (1953c). This type carries the same determining phage as type $D_{1}$ and no other phage has hitherto been isolated from it. Stored cultures of type $D_{4}$ show a single-step change to type $D_{1}$ in a proportion of cells. The type $D_{1}$ so formed is still carrying the determining phage carried by the original culture. Thus, the change in type $D_{4}$ that yields type $D_{1}$ must occur in the non-lysogenic precursor of $D_{4}$. This change might yield type $A$ 
were it not for the presence of phage $d_{1}$, which limits the expansion of Vi-phage sensitivity of the organism to the pattern of type $D_{1}$.

Typhoid cultures sent for phage typing not infrequently react as degraded Vi strains. In some cases the patient from whom they were isolated may have been excreting a mixture of a specific type and its degraded variant. For this reason it is desirable to pool a number of colonies, from primary platings if possible, when preparing a culture for despatch to an enteric reference laboratory. The chances of the specific type being represented in the culture are thereby greatly increased.

It is not uncommon for patients to be infected with strains that present a uniformly degraded reaction. This may not be associated with any further identifiable feature, but in some instances temperate phages can be isolated from these strains. Lysogenization of type A with these phages will modify the reaction of the culture so as to resemble that of the strains from which the phages were isolated (Anderson, unpublished observations ; Ferguson, Juenker, and Ferguson, 1955). In other words, the phages have a limited type-determining function. As the sensitivity of the newly lysogenized type A cultures to the $\mathrm{Vi}$ typing phages is of a lower order than that of the original type $\mathrm{A}$, it is obviously incorrect to refer to them, or to the cultures from which the temperate phages were isolated, as degraded strains, because this implies a change from a higher to a lower specificity, which may be the reverse of what has occurred. For the moment, however, the designation "degraded Vi strains" is applied to all cultures showing a wide sensitivity spectrum to the Vi-typing phages. It should eventually be possible to arrive at a more precise designation for some of these strains. Obviously, the isolation of temperate phages such as those just described (which might, perhaps, be called "semi-determining" phages) offers an additional method of characterization of strains.

It may be mentioned here that the expression degradation refers only to a particular type of lytic pattern with the Vi-typing phages. It does not imply that any antigens are lost or that the pathogenic properties of the strains concerned are in any way diminished.

Cultures resistant to the Vi-typing phages (that is, untypable $\mathrm{Vi}$ strains) may carry identifiable type-determining phages. Such a finding sometimes indicates that in preparing typing phages for such cultures it is advisable to start with hostrange mutants of Vi-phage II. The identity of the type-determining phage indicates the nature of the mutant required. For example, the isolation of phage $d_{1}$ suggests that the adaptation should start from phage $D_{1}$; and the isolation of phage $d_{6}$ suggests that the adaptation should begin with phage $D_{6}$. Phages $D_{1}$ and $D_{6}$ have been proved to be host-range mutants of Vi-phage II (Anderson and Felix, 1953a; Anderson and Fraser, 1955). The former can overcome the obstacle to multiplication erected by the determining phage $d_{1}$; the latter performs a similar function in the presence of phage $d_{6}$. Thus, the amount of change required to convert each mutant into a typing phage for types that owe their specificity in part to the respective temperate phages $d_{1}$ and $d_{6}$ is smaller than the change that would be necessary in phage A. This subject has been fully discussed in a recent publication (Anderson, 1956).

Type Specificity in Salmonella paratyphi B.-It has been shown (Scholtens, 1950 ; Felix and Callow, 1951 ; Hamon and Nicolle, 1951 ; Nicolle, Hamon, and Edlinger, 1951 ; Scholtens, 1952, 1955a, 1956) that all the phage types of $S$. paratyphi B are lysogenic. Each type carries a characteristic phage or phages, and indeed, as has been pointed out earlier, some of the typing phages of the paratyphoid B typing scheme are identical with the phages carried by particular types. It has been found that the specificity of the phage types of $S$. paratyphi B is dependent upon the characteristic lysogenicity of each type. Hamon and Nicolle (1951) were able to convert type 1 into type 2 with a phage isolated from type 2 , and type $3 a$ into type B.A.O.R. with a phage isolated from B.A.O.R. Similarly, the phages carried by types Beccles, Taunton, and Dundee converted type $3 \mathrm{a}$ into the respective types from which the phages had been isolated. In this scheme, then, some phages carry out the dual function of phage typing and of type determination.

Scholtens (1950) has suggested typing the paratyphoid $B$ bacillus by the identification of the characteristic phages carried by each type, in a manner analogous to that proposed by Boyd (1950) and Boyd et al. (1951) for S. typhimurium. Scholtens's suggestions were further developed in later papers (Scholtens, 1955a, 1956). There is no theoretical objection to type distinction by the identification of the temperate phages carried by each type. The practical use of the method is limited by the ease with which phage identification can be carried out. If this is a simple matter entailing, for example, no more than the determination of the plaque characters and host range of the phages concerned, or of the specific type changes brought about by the lysogenization of indicator 
strains, the method presents no great technical difficulties, although it is much slower than the method of Felix and Callow. If, however, it also requires serological identification of the phages and the determination of their physical properties, the method is too time-consuming for routine use. However, it can always be used as an ancillary method of typing, in addition to the routine technique of application of typing phages, as we have indicated in the case of S. typhi.

Salmonella typhimurium.-Work so far carried out has indicated that type specificity in S. typhimurium is due to similar causes to that of $S$. paratyphi B and S. typhi, that is, the presence of characteristic resistance patterns controlled by lysogenicity (Callow, to be published). As we have pointed out above, Boyd (1950) suggested the use of temperate phage identification as a method of typing $S$. typhimurium. This method was used with success by Boyd et al. (1951). Some of the phages identified by Boyd's technique may have a type-determining function in relation to the reactions of cultures of $S$. typhimurium with the routine typing phages used in the method of Felix and Callow, and in such instances the two typing methods examine different facets of the same problem.

\section{Conclusions}

The Vi-phage typing scheme for Salmonella typhi is the ideal of its kind. The typing preparations are all prepared from one phage and, when used in R.T.D., present a specificity of host range that makes type diagnosis a relatively simp'e matter. The international use of centrally standardized preparations, and the free exchange of information through the medium of the International Committee for Enteric Phage Typing by laboratories using the method throughout the world, have placed this technique on a sound footing and provided epidemiologists with a tool of unparalleled precision for tracing the sources of typhoid fever.

The paratyphoid $B$ phage typing scheme, because it employs a battery of largely unadapted and frequently dissimilar phages, is less specific. Nevertheless, in the hands of experienced workers it has shown a reliability approaching that of the typhoid Vi-typing scheme. Here again, international standardization has resulted in a high degree of accuracy and is contributing to the control of paratyphoid fever in many countries.

The phage typing of Salmonella typhimurium has a reliability similar to that of $S$. paratyphi B. This method is not internationally standardized at present, but the need to do so is no less than in the cases of the typhoid and paratyphoid B bacilli.
The free international exchange of foodstuffs, many of which are liable to contamination with $S$. typhimurium at any stage of their preparation from the animal to the consumer, makes it a matter of growing importance that phage type identification should be as practicable in the country exporting the food as in that importing it. The recent articles by Hobbs and Smith (1955), Newell (1955a, b), Newell et al. (1955), and Smith and Hobbs (1955) concerning the presence of $S$. paratyphi B, S. typhimurium and other salmonellas in Chinese egg products are sufficient proof of this.

Finally, it must be emphasized again that phage typing is of no value unless full use is made of the highly accurate information it provides. Thus, wherever it is used it should be part of a chain of which the clinician, the laboratory worker, the epidemiologist, and the public health authorities are all indispensable links.

\section{REFERENCES \\ Enteric Phage Typing}

Anderson, E. S. (1951). J. Hyg. (Lond.), 49, 458.

(1955a). Nature (Lond.), 175, 171.

(1955b). J. gen. Microbiol., 12, 379.

(1956). Ibid., 14, 676.

and Felix, A. (1952). Nature (Lond.), 170, 492.

(1953a). J. gen. Microbiol., 8, 408.

(1953b). Ibid., 9, 65.

- (1953c). Proc. 6th. int. Congr. Microbiol., Rome, vol. 3, p. 462.

- and Fraser, A. (1955). J. gen. Microbiol., 13, 519.

- (1956). Ibid., 15. In the press.

Banker, D. D. (1955). Nature (Lond.), 175, 309.

Bertani, G., and Weigle, J. J. (1953). J. Bact., 65, 113.

Blasi, R. de, and Buogo, A. (1952). Riv. ital. Igiene, 12, 16.

Boyd, J. S. K. (1950). J. Path. Bact., 62, 501.

- Parker, M. T., and Mair, N. S. (1951). J. Hyg. (Lond.), 49, 442.

Bradley, W. H. (1943). Brit. med. J., 1, 438.

Brandis, H. (1955). Zbl. Bakt., 1. Abt. Orig., 164, 149.

Cherry, W. B., Davis, B. R., and Edwards, P. R. (1953). Amer. J. publ. Hlth, 43, 1280.

Cockburn, W. C., Jameson, J. E., and Fenton, J. (1951). Mon. Bull. Minist. Hlth (Lond.), 10, 43.

Craigie, J. (1940). Proc. 3rd int. Congr. Microbiol., New York, 1939 , p. 296.

(1942). Canad. J. publ. Hlth, 33, 41.

(1946). Bact. Rev., 10, 73.

and Brandon, K. F. (1936). J. Path. Bact., 43, 233.

and Felix, A. (1947). Lancet, 1, 823.

and Yen, C. H. (1938a). Canad. J. publ. Hlth, $29,448$.

- (1938b). Ibid., 29, 484.

Cruickshank, J. C. (1947). Mon. Bull. Minist. Hlth (Lond.), 6, 88.

Desranleau, J. M. (1947). Canad. J. publ. Hlth, 38, 343.

and Martin, I. (1950). Ibid., 41, 128.

Dhayagude, R. G., and Banker, D. D. (1951). Indian J. med. Res., $39,1$.

Edwards, P. R., Bruner, D. W., and Moran, A. B. (1948). J. infect. Dis., 83, 220.

Eörsi, M. (1956). Acta microbiol. (Bp.), 3, 285.

Felix, A. (1943). Brit. med. J., 1, 435. (1944). Brit. med. Bull., 2, 269.

(1949). Proc. 4th int. Congr. Microbiol., Copenhagen, 1947, p. 355.

(1950). 5th int. Congr. Microbiol., Rio de Janeiro. Abstracts of papers, p. 242.

(1951). Brit. med. Bull., 7, 153. 
Felix, A. (1955). Bull. Wld Hlth Org., 13, 109.

- (1956). J. gen. Microbiol., 14, 208.

and Anderson, E. S. (1951a). Nature (Lond.), 167, 603. (1951b). J. Hyg. (Lond.), 49, 349.

and Callow, B. R. (1943). Brit. med. J., 2, 127 (1951). Lancet, 2, 10.

- and Pitt, R. M. (1934). Ibid., 2, 186.

Ferguson, W. W., Juenker, A., and Ferguson, R. A. (1955). Amer. J. Hyg., 62, 306.

Hamon, Y., and Nicolle, P. (1951). Ann. Inst. Pasteur, 80, 496.

Hobbs, B. C., and Smith, M. E. (1955). J. appl. Bact., 18, 471.

Jacob, F., Lwoff, A., Siminovitch, L., and Wollman, E. (1953). Ann. Inst. Pasteur, 84, 222.

Kennedy, J., and Payne, A. M.-M. (1950). Mon. Bull. Minist. Hlth (Lond.), 9, 168.

King, G. J. G. (1944). Ibid., 3, 84.

Kristensen, M. (1938). J. Hyg. (Lond.), 38, 688.

- and Henriksen, H. C. D. (1926). Acta path. microbiol. scand., 3,551 .

Lendon, N. C., and Mackenzie, R. D. (1951). Mon. Bull. Minist. Hlth (Lond.), 10, 23.

Lie Kian Joe (1949). Docum. neerl. indones. Morb. trop., 1, 145.

Lilleengen, K. (1948). Acta path. microbiol. scand., Suppl. 77.

- (1950). Ibid., 27, 625.

- (1952). Ibid., 30, 194.

Lisbonne, M., and Carrère, L. (1922). C. R. Soc. Biol. (Paris), 86, 569. - (1923). Ibid., 88, 724.

Luria, S. E., and Delbrück, M. (1943). Genetics, 28, 491.

- and Human, M. L. (1952). J. Bact., 64, 557.

Lwoff, A. (1953). Bact. Rev., 17, 269.

Martin, P. H. (1947). Mon. Bull. Minist. Hlth (Lond.), 6, 148.

Medical Research Council (1947). Spec. Rep. Ser. med. Res. Coun. (Lond.), No. 260.

Ministry of Health (1954). Report for the year ended December 31, 1953, Part 2, p. 83 . London.

Moore, B. (1948). Mon. Bull. Minist. Hlth (Lond.), 7, 241.

- (1950). Ibid., 9, 72.

Newell, K. W. (1955a). Ibid., 14, 146.

- (1955b). J. appl. Bact., 18, 462.

Hobbs, B. C., and Wallace, E. J. G. (1955). Brit. med. J., 2, 1296.

Nicolle, P., Hamon, Y., and Edlinger, E. (1951). Ann. Inst. Pasteur, 80, 479.

--1 (1953). Biol. méd., 42, 437.

- Jude, A., and Buttiaux, R. (1950). Ann. Inst. Pasteur, 79, 246. Pavlatou, M., and Diverneau, G. (1954). Ibid., 87, 493.

Olitzki, A. L., and Shel ıbsky, M. (1949). Report Research Council of Israel, Jerusalem, 1949.

- and Strauss, W. (1948). Harefuah, 34, 107.

Pavlatou, M., and Nicolle, P. (1953). Ann. Inst. Pasteur, 85, 185.

Perei ia, M. S., and Blaxland, J. D. (1955). Mon. Bull. Minist. Hlth. (Lond.), 14, 52.

Rische, H. (1954). Z. ges. inn. Med., 9, 896.

- (1955). Z. ges. Hyg., 2, 110.

Sampaio, A., and Figueiredo, A. (1955a). Bगl. Serv. Saúde púb!., 2 , No. 2, p. 15 .

(1955b). Ibid., 2, No. 2, p. 33.

Scholtens, R. T. (1936). J. Hyg. (Lond.), 36, 452.

(1950). Antonie v. Leeuwenhosk, 16, 256

(1952). Ibid., 18, 257.

(1955a). J. Hyg. (Lond.), 53, 1.

(1955b). Ann. Inst. Pasteur, 83, 216.

- (1956). Antonie v. Leeuwenhoek, 22, 65.

Sertic, V., and Boulgakov, N. A. (1936). C. R. Soc. Biol. (Paris), 122, 35.

Sicca, G. T., and D'Amelio, V. (1953). Riv. Ist. sieroter. ita!., 28, No. 6.

Smith, H. G. (1950). Mon. Bull. Minist. Hlth (Lond.), 9, 126.

Smith, H. W. (1951a). J. gen. Microbiol., 5, 458.

- (1951b). Ibid., 5, 472.

- (1951c). Ibid., 5, 919.

Smith, M. E., and Hobbs, B. C. (1955). Mon. Bull. Minist. H/th (Lond.), 14, 154.

Thomson, S. (1953). Ibid., 12, 187.

Warren, S. H., and Goldie, W. (1945). Ibid., 4, 179.

Wallace, W. S., and Mackenzie, R. D. (1947). Ibid., 6, 32.

Weigle, J. J., and Bertani, G. (1953). Ann. Inst. Pasteur, 84, 175.

\section{PHAGE TYPING OF STAPHYLOCOCCUS AUREUS}

\section{Historical}

The first workers to use bacteriophages in an attempt to distinguish strains of staphylococci seem to have been Williams and Timmins (1938), who tested broth cultures for their susceptibility to the series of phages isolated by Burnet and Lush (1935). However, the basis of the current methods was laid by Fisk's (1942) demonstration that many strains of Staph. aureus are lysogenic, that is, they carry bacteriophages. These phages are able to lyse some but not all other strains. By testing staphylococci with a number of the phages a "susceptibility pattern" could be recognized, and by comparison of the patterns similarity or dissimilarity of two strains could be determined. Fisk recognized his lysogenic staphylocosci by the method of " cross-culture," in which spot inocula of a number of different strains were applied to a plate previously spread with one or more strains. Plaques of lysis on the spot area (Fig. 6) indicated that a phage was carried by either the applied or the lawn culture. Fisk maintained his phages in mixed culture with the staphylococci on which they had been propagated and freed them from the cocci immediately before use by treatment with the quaternary ammonium disinfectant, zephiran. Typing was carried out by spotting these phage

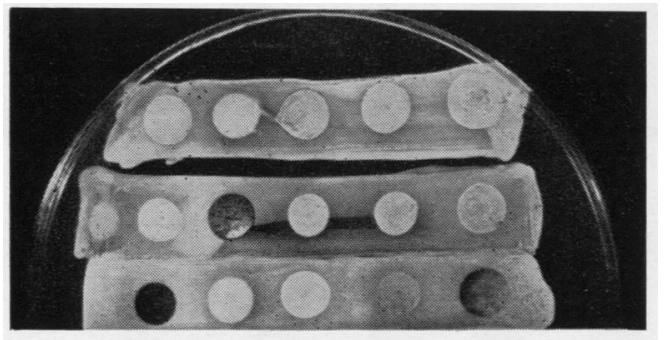

FIG. $6 a$.

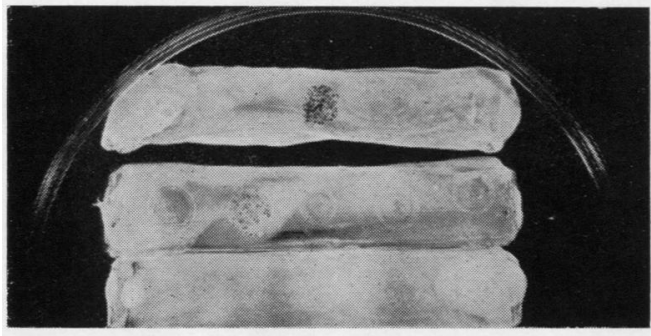

FIG. $6 b$.

Fig. 6.-Cross-culture tests for identification of lysogenic strains; (a) shows the result of inoculating whole culture on to areas spread with the test culture, and (b) the result of inoculating culture supernatants. 
preparations on to an agar culture plate previously spread with the culture under test. Despite its obvious disadvantages the method was successfully employed in two small epidemiological studies (Fisk and Mordvin, 1944; McClure and Miller, 1946).

Wilson and Atkinson (1945) demonstrated that the phages could be separated from the cocci by filtration and that the phage filtrates were stable; they isolated and tested numerous phages from different strains of staphylococci, and obtained still more by adaptation of these phages to new propagating strains. In their 1945 paper they reported the use of a total of 18 phages, 11 isolated by Fisk's cross-culture method and seven by adaptation. They also defined 21 different phage types of staphylococci-based on a reaction of confluent lysis produced by single phages or by groups of three or four phages acting on the organism under test. The definition of clear-cut types by phage susceptibility patterns has not been adopted to any great extent in subsequent work, but apart from this the methods devised by Wilson and Atkinson have been refined rather than altered in the subsequent 10 years.

In 1946 a Staphylococcus Reference Laboratory was set up for the Public Health Laboratory Service under Dr. V. D. Allison, who directed it until 1948 , and it is largely on the basis of the experience of this laboratory that the present review is written. A full account of the methods used was published in 1952 (Williams and Rippon), and the description given below is, unless otherwise indicated, based on that paper or on subsequent unpublished observations.

Throughout this paper the term "staphylococcus" refers to coagulase-positive strains; the phages are, almost without exception, unable to attack coagulase-negative cocci.

\section{The Phages of the Staphylococi}

Fisk's early work demonstrated that many staphylococci are lysogenic. This finding was amplified by Rountree (1949a), who showed that some cultures might yield several different strains of phage. Rountree (1949b) also found that the phages could stimulate the production of neutralizing antibodies when injected into rabbits, and that such antisera could be used as a basis for classifying the phages. A total of eight different serological groups of phages acting on coagulasepositive staphylococci are now recognized.

Rippon (1956) has described two main divisions of the staphylococcal phages: the polyvalent phages, and those of restricted host-range. The former have been isolated principally from sewage $\underline{\vec{O}}$ and ordinarily attack all coagulase-positive staphylococci ; they belong to the serological $\stackrel{5}{?}$ groups known as $\mathrm{D}, \mathrm{G}$, and $\mathrm{H}$, and are exemplified by the well-known " $K$ " phages. They are 흠 not generally used in typing although the only $\frac{\text { के }}{\overrightarrow{0}}$ three representatives of Group $G$ were so used $\stackrel{\mathbb{Q}}{\varrho}$ by Wallmark (1949) and by Wahl and Lapeyre- ळ Mensignac (1950). The phages of restricted host- $\vec{\circ}$ range are those used in typing. They are typical "symbiotic" or "temperate" phages, isolated $\vec{\omega}$ from normal lysogenic strains, and able to re- $\frac{\Omega}{\circ}$ establish the lysogenic state when they infect new ? strains under appropriate conditions. They can be $c$ classified on the basis of their host-range on a set of indicator strains (see below), and, by serum neutralization tests, into the serological groups $\mathrm{A}$, 을 B, F, and L (Rippon, 1956).

The staphylococcal phages have been studied from a general biological aspect much less than the phages of $E$. coli or $S$. typhi, but they have $\vec{E}$ been shown to resemble the more fully studied $\triangle$ phages in several ways, for example, their "tad- D pole " shape and attachment to the coccus by their "tail" (Farrant and Rountree, 1953), their need for calcium ions (in some instances replaceable by magnesium) for adsorption to the cocci (Smith, $\stackrel{\mathbb{Q}}{\Omega}$ 1948a ; Rountree, 1951), their ability to establish $\overrightarrow{\vec{P}}$ the lysogenic state, and the protective effect of $\frac{3}{3}$ such lysogenicity against subsequent lysis by the $\bar{\tau}$ same phage (Smith, 1948b). The full extent to which the carried phages determine the phage susceptibility of staphylococci is not, however, known and there is no structural scheme available $\frac{5}{3}$ comparable with that described for the S. typhi types.

\section{Technique of Phage Propagation and Typing}

Phage Propagation.-The phages may be propa- $D$ gated by growing them on cultures of the appropriate staphylococci on the surface of agar plates or in $\bar{N}$ broth. In the first method a plate (commonly $15 \mathrm{~cm}$. in diameter) poured with soft nutrient agar to a depth $\mathrm{N}$ of $5 \mathrm{~mm}$. is spread with a young broth culture of $N$ the staphylococcus; a phage filtrate is then spread $\frac{\omega}{\sigma}$ over it at a dilution calculated from preliminary experiments to give nearly confluent lysis after incubation at $30^{\circ} \mathrm{C}$. overnight. The phage can be recovered

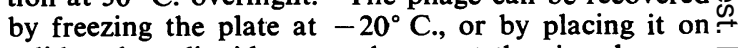
solid carbon dioxide; on subsequent thawing the agar $\square$ disintegrates and the expressed fluid contains the $\bar{P}$ phage. The phage yield is highest when lysis of the staphylococcal lawn is not quite complete.

In the second and better method, the phage is propa- $\varrho$ gated on staphylococci growing in a fluid medium. Wahl and Lapeyre-Mensignac (1950) employed a glucose peptone water, but it is our practice to use्d 
nutrient broth without any enrichment, except that 0.1-1.0 g./litre calcium chloride may be needed by some of the phages (those of serological group B) and with some samples of broth. The optimum proportion of staphylococcus and phage in the inoculum has to be determined by test, but a final $1 / 100$ dilution of a two-hour broth culture of the staphylococcus and between 10 times and 1/10 the R.T.D. (see below) of phage usually proves satisfactory.

In either case the phage is freed from the staphylococci by filtration of the fluid through a gradocol or molecular membrane filter, or, with reasonably large quantities of a high-titre phage, a Seitz pad. The staphylococcus typing phages are inactivated at a temperature below that which kills the cocci, and heat cannot therefore be used for ridding the lysate of living cocci. Wahl and Blum-Emerique (1949) have employed thymol to sterilize the lysate, but this method has the disadvantage that the thymol makes high concentrations of the lysate inhibitory to all staphylococci, and yet such concentrations may often be useful for testing insensitive strains of staphylococci.

Phage Testing.-As with all diagnostic reagents, the thorough testing of new batches of phage is of immense importance in ensuring reproducibility of results. First, it is important that the lysate should be of sufficient strength : the titre is ordinarily defined, as in the phage typing of the enteric organisms, as the dilution that just produces confluent lysis when a single drop is applied to a lawn of the propagating strain of staphylococcus: this is the routine test dilution (R.T.D.). With most of the typing phages filtrates having an R.T.D. of $1 / 10,000$ to $1 / 100,000$ can be obtained by either broth or agar propagation and ordinarily filtrates with an R.T.D. of less than $1 / 1,000$ are not used.

Second, it is important that the phage should have the standard "lytic spectrum," that is, it should lyse the proper range of the standard set of 22 staphylococcal strains that are used for testing; and that the titre on the susceptible strains should be, within reasonable limits, a standard fraction of the titre on the propagating strain. For example, phage $3 \mathrm{~A}$ should lyse the staphylococcal strains P.S. 3B, P.S. 3C, P.S. 51 at 100,10 , and 1,000 times respectively the concentration giving equivalent lysis of its propagating strain P.S. 3A, and should fail to lyse any of the other 18 test strains. The undiluted filtrate of each new batch of phage is therefore tested first on the complete set of 22 indicator staphylococci; dilutions of the filtrate are retested on any strains lysed by the strong filtrate.

The phages are ordinarily used at two dilutions : the routine test dilution defined above, and, for staphylococci not lysed by this dilute phage, at 1,000 times stronger than this dilution. It is our practice to check the routine phage dilutions twice weekly by applying a drop of each to agar plates previously spread over a small area with the appropriate propagating strain. Any filtrate that fails to produce strong lysis of its strain is replaced.

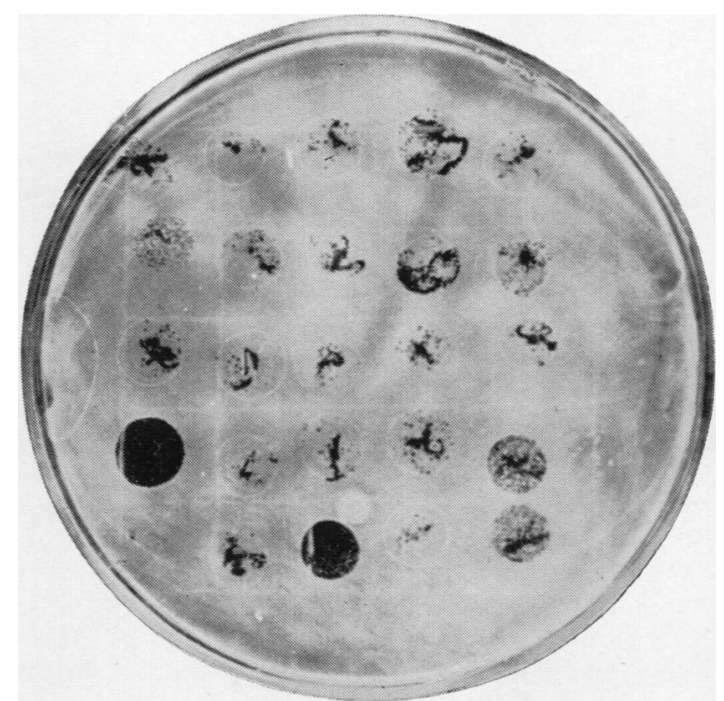

FIG. 7.-Non-specific lysis due to phage libe:ated by staphylococci carried over from the previous culture; this can be avoided by care in the application of the phage drops to the plates.

Phage Preservation.-The phages have been found to keep well in the broth of the original filtrate when stored at $+4^{\circ} \mathrm{C}$. Provided that the dilutions to the working strength are also made in broth, these retain their activity for several weeks at $+4^{\circ} \mathrm{C}$. The lysates are also readily preserved by freeze-drying without the addition of any serum, etc.

Supply of Phages.-Central supply of staphylococcus phage filtrates has not been organized, but an attempt has been made to ensure so far as possible that all workers use phages from one stock, and that all have a set of the standard testing strains. Further collaboration and exchange of information is arranged through the members of the Subcommittee on Bacteriophage Typing of Staph. aureus of the International Bacteriological Nomenclature Committee.

Typing of Staphylococci.-In the Colindale routine, the staphylococci for testing are inoculated into nutrient broth and incubated at $37^{\circ} \mathrm{C}$. for four to five hours, or sufficiently long to give a moderate turbidity. Four-inch $(9 \mathrm{~cm}$.) Petri dishes, having a grid of 25 half-inch $(1.3 \mathrm{~cm}$.) squares outlined on their surface, are poured to a depth of about $5 \mathrm{~mm}$. with a nutrient agar (Difco dehydrated nutrient broth $20 \mathrm{~g}$. per litre set with about 10 to $12 \mathrm{~g}$. agar per litre) and dried until free of surface moisture. They are then flooded with 1-2 ml. of the culture. The excess is pipetted off and the plates are allowed to dry. Drops of the phage filtrates are then applied, with fine Pasteur pipettes, to the agar over the centre of each of the ruled squares. The phages are applied in a prescribed order so that each square always corresponds to a particular phage. The drops are "touched off" from the end of the pipette, but care has to be taken to avoid touching the agar with the 
pipette, since this may lead to transfer of staphylococci from one plate to the next; the staphylococci may carry phages of their own that are capable of lysing the cocci on the next plate, and such phages may then lead to the appearance of non-specific plaques of lysis (Fig. 7).

When the phage drops have dried the plates are incubated at $30^{\circ} \mathrm{C}$. for 18 hours.

The plates are examined with a hand lens $(\times 4-\times 6)$ under oblique transmitted illumination and the degree of lysis produced by each phage is recorded. The practice at Colindale is to regard all drop areas having more than about 50 plaques as showing strong lysis $(++)$; those with $40-50$ plaques as moderate $(+)$, and those with less than 20 as weak $( \pm)$. When phage filtrates stronger than the routine test dilution are used, additional symbols to denote confluent lysis $(++++)$ and strong lysis with heavy secondary growth $(t+)$ are employed.

The technique just described appears to have been followed with only minor variations by most workers. Blair and Carr (1953) were able to place 30 instead of 25 drops on a $9 \mathrm{~cm}$. plate. Wahl and Fouace (1954) considered that plates should be examined after 48 as well as after 24 hours.

However, Goldberg (1954) has introduced a considerable technical modification in that he uses small agar cells formed in wax rings on microscope slides, one cell for each phage; he claims that this has the advantage that the phage drops can be applied more quickly since there is no chance of their running together, but in other respects the method seems unduly complicated.

\section{Use of Different Phage Dilutions}

Following the practice adopted in the typhoid $\mathrm{Vi}$ phage typing scheme, the staphylococcus typing phages have generally been employed at their routine test dilution (see above). About one-third of all strains of staphylococci encountered are, however, insensitive to this dilution of all the commonly employed 20-30 phages. For characterizing these strains we have the alternatives of testing with a wider range of phages or of using the usual set of phages at a greater concentration; in our experience the latter has been by far the most rewarding, although the former has had its notable successes (see Rountree and Freeman, 1955 ; Parker, Tomlinson, and Williams, 1955). During the last few years we have routinely employed the phages at 1,000 times the concentration of the R.T.D. on strains not lysed by any phage at R.T.D. ; only about $8 \%$ have then failed to show strong lysis with at least one phage.

The major disadvantage of using the phage filtrates at high concentration is the inhibitory effect of such strong filtrates on some strains. This inhibition, which is also seen with some other phage cell systems (see section on $S$. typhi), may give rise to areas of clearing in the typing plates that are distinguishable from confluent lysis only by further tests with a series of phage dilutions : it is then found that at no dilution

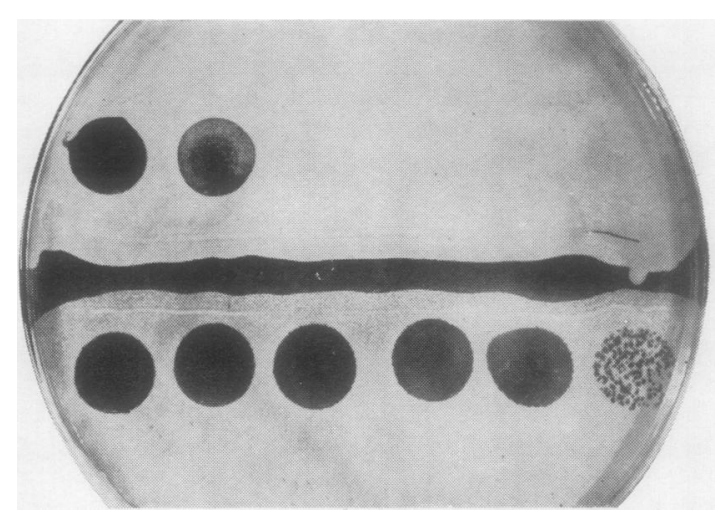

FIG. 8.-Inhibition of the test staphylococcus produced by high concentration of phage filtrate. The top half of the plate was inoculated with ten-fold dilutions of a phage that was unable to multiply on the staphylococcus; the areas of confluent or semiconfluent "lysis" are due to inhibition of growth, and discrete plaques are not seen at any dilution. Below is a normal phage titration.

are discrete plaques to be seen (Fig. 8). This inhibitory effect is much less commonly seen with phages used 100 times stronger than the R.T.D. than at 1,000 times, and Wahl and Fouace (1954) have therefore suggested that such a concentration should be employed in all phage typing to the exclusion both of the R.T.D. and the R.T.D. $/ 1,000$. No one else has yet reported on the value of this suggestion; in our experience it has one serious disadvantage-namely, of giving very much more complex lytic patterns for the strains sensitive to the R.T.D. Nevertheless, the R.T.D. is an arbitrary level of dilution, and possibly some variation might be useful.

\section{Selection of Typing Phages}

There is no difficulty in isolating from staphylococci phages of the general character of those used $\frac{0}{3}$ for typing. It is also often possible to adapt them 0 to grow on other strains and thus usually to change the range of staphylococci that they will attack, but the adapted phages are not generally typespecific. There would be advantages in using a $\sigma$ set of type-specific phages, such as those derived $N$ from the typhoid Vi-phage II-type-specific in that one strain of staphylococci would be attacked by one phage and one only. However, despite several attempts, no parent phage has been found that will $\frac{\mathrm{C}}{\bar{D}}$ give rise to adapted progeny of the sort given by Vi-phage II. Nor has a search for naturally- $\square$ occurring type-specific phages proved very profit-우 able, though some phages, such as 71 and 80 , are frequently the only phages able to lyse particular strains.

With the set of phages that was employed by Wilson and Atkinson (1945), and the modified setso응 
used since, most staphylococci are lysed by more than one phage. Thus of 229 strains lysed by one or more phages, 60 were lysed strongly by two phages, 27 by three, and 24 by four or more phages ; in many cases the strains were also lysed less strongly by other phages. Typing with these phages is therefore necessarily based on the recognition of patterns.

There is, however, another side to this. If the numerous apparently distinct phage patterns really represent different types of staphylococci, the number of different types is so great that it would be impracticable to carry the necessarily wide range of type-specific phages, even if they were available. To do so would be like attempting to carry a complete range of sera specific for each of the 400 or more Salmonella types. It may be that the current method of using a small number of phages to define a large number of patterns is the better. Type-specific phages are more likely to be of use in the extended investigation of particular hospital epidemics and the like than in a country-wide typing service.

The set of phages commonly employed, therefore, has been chosen to give a reasonable compromise between $(a)$ the relative non-specificity needed to yield some lysis on almost all strains with a set of no more than 20 to 25 phages, with (b) the greatest possible independence of action between the phages so that no two phages are constantly found together in lytic patterns, and (c) reasonable specificity so that the patterns of lysis do not comprise reactions with too many phages.

A basic set of 19 phages was agreed to at a meeting of the provisional International Subcommittee on Phage Typing of Staphylococci held in Rome in 1953 (unpublished), and this set has been adopted in many laboratories in different countries (Table VIII). Where it is desired to use more than about 25 phages it is convenient to mix some of the less commonly active filtrates into pools.

Our present practice is to test all staphylococci first with a basic set of 21 phages, two-71 and 80 -having been added since 1953 ; any strains that show no strong lysis with any of these 21 phages are retested both with the more concentrated (R.T.D./1,000) phage dilutions and with pools of the additional phages given in Table VIII. A further test is carried out with the individual phages of any pool that produces lysis, but the reactions of the phages in the pools are only reported if none of the basic set of phages lyses the strain, even at the stronger concentration.
TABLE VIII

TYPING PHAGES EMPLOYED IN THE STAPHYLOCOCCUS REFERENCE LABORATORY, COLINDALE, APRIL, 1956

\begin{tabular}{|c|c|c|c|c|c|c|c|}
\hline \multirow{2}{*}{$\begin{array}{l}\text { Lytic } \\
\text { Group }\end{array}$} & \multicolumn{3}{|c|}{ Phages in Basic Set } & \multicolumn{4}{|c|}{ Phages in Pools } \\
\hline & $A^{*}$ & B & $\mathbf{F}$ & $\mathbf{A}$ & B & $\mathbf{F}$ & $\mathbf{L}$ \\
\hline $\mathbf{I}$ & - & $\begin{array}{l}29,52, \\
52 \mathrm{~A}, 79 \\
80\end{array}$ & - & - & - & - & - \\
\hline III & $\begin{array}{c}3 \mathrm{~A}, 3 \mathrm{~B}, 3 \mathrm{C} \\
6,7,42 \mathrm{E}, \\
47,54,70, \\
73,75\end{array}$ & $\begin{array}{c}55,71 \\
53-\end{array}$ & $\overline{7}$ & $\begin{array}{l}51 \\
42 \mathrm{~B}, 47 \mathrm{~B}, \\
47 \mathrm{C}, 75 \mathrm{~A}, \\
75 \mathrm{~B}\end{array}$ & $31 \mathrm{~B}, \overline{5} 2 \mathrm{~B}$ & $\overline{7}$ & 二 \\
\hline $\begin{array}{c}\text { IV } \\
\text { Unclus- } \\
\text { sifiable }\end{array}$ & = & = & $42 \mathrm{D}+$ & $\begin{array}{l}42 \mathrm{~F} \\
47 \mathrm{~A}, 57 \\
78,81\end{array}$ & $\begin{array}{l}31, \overline{42} \mathrm{C} \\
44,44 \mathrm{~A} \\
69\end{array}$ & $\overline{58}$ & $\overline{18}$ \\
\hline
\end{tabular}

* Columns A, B, F, and $L$ indicate the serological group of the phages. $\uparrow$ The original phage 42D was se:ological Group $B$, but a Group $F$ variant that appeared in the stock at Colindale appears to be rather more specific (Rippon, 1954).

During recent years we have found that about $65 \%$ of strains are typable with the phages at R.T.D. and that about a further $27 \%$ are typable with the strong phages. The percentage untypable with the basic set of phages at any dilution has been about 8 ; of these one-tenth to onehalf have been typable with extra phages.

\section{Isolation of New Phages}

Two methods can be used for the isolation of a new phage, for example, for characterizing strains that are not lysed by any of the existing phages. First an attempt may be made to "adapt" one of the existing phages; undiluted filtrates of a number of phages are spotted on to a plate spread with the untypable strain. Or, second, one can try to isolate a new phage from lysogenic staphylococci by the method of cross-culture. This is conveniently carried out by spotting the supernatant fluid from young broth cultures of the putative lysogenic strains on to a plate spread with the untypable strain; phage particles are usually present in the supernatant fluid of cultures of lysogenic strains and the use of the supernatant fluid rather than whole cultures has the advantage of reducing the amount of growth of the donor culture, and therefore facilitates interpretation (Fig. 6b).

If plaques develop, in either case, the phage can be isolated by touching the plaque and adjacent staphylococcal growth with a platinum wire, transferring to about $1.0 \mathrm{ml}$. of nutrient broth, and spreading the broth on a fresh agar plate. The growth usually shows numerous plaques, which may be purified and built up to a usable titre by repetition of the same process.

Most of the new phages thus isolated are rather similar to one or other of the phages already in 

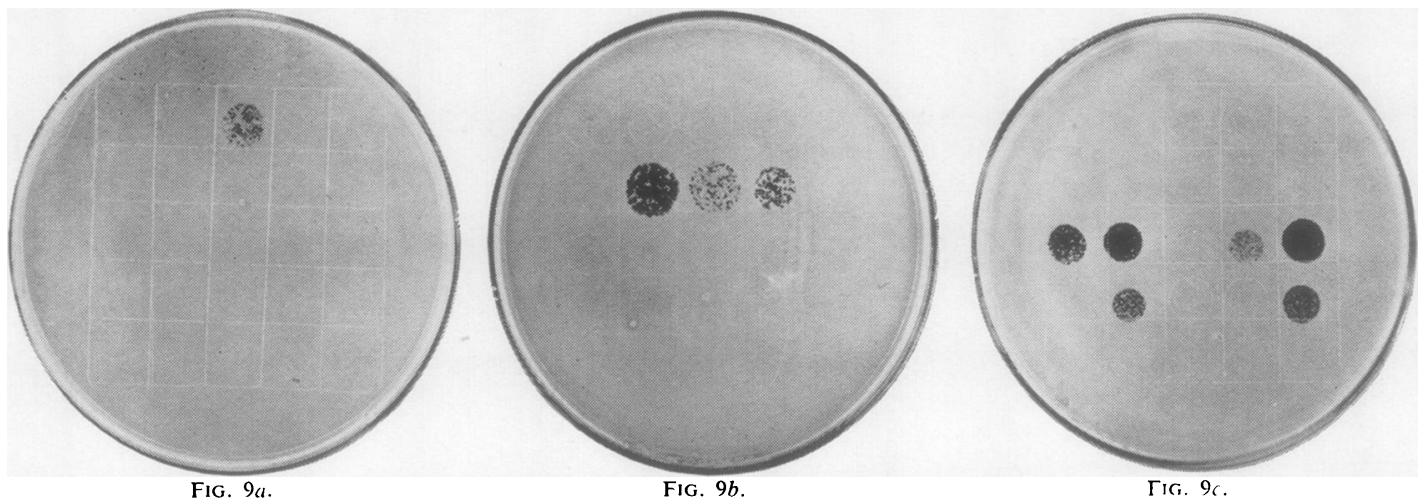

FIG. 9. - Three typical phage susceptibility patterns. $(a)++$ lysis by phage $52 \mathrm{~A}$ alone, $(b)+-$ lysis by phages $3 \mathrm{~A}, 3 \mathrm{~B}$, and $3 \mathrm{C}$. (c) - lysis by phages $6,7,47,53,70$, and 77 with $\ldots$ lysis by phage $42 \mathrm{E}$ (centre of third row).

the typing set. They need to be tested, first, by the routine described for testing new batches of phage, and then by inclusion in the routine typing set for long enough to assess their usefulness in the identification of recently isolated strains.

The process of adaptation is almost certainly in some cases one of selection of one phage out of a mixture; it is known that the carried phages of the propagating strains of staphylococi are usually present in low titre in the typing filtrates and the new phage may be one of these that has been selected by the new strain. Rountree (1949b) regards this as the origin of phage $42 \mathrm{C}$.

In other cases the adaptation is probably a real change induced in the phage particles under the influence of the new host. Rippon (1954) and Rountree (in the press) have shown that such phenotypic modification-readily reversible-can occur.

\section{Interpretation of Typing Results}

Despite the suggestions by Wilson and Atkinson (1945), and also by Wallmark $(1949,1954)$ that the different phage patterns could be used to define types, most workers have found that the patterns are too numerous, and the degree of overlap between them too great for this to be practicable (see Williams and Rippon (1952) for discussion of this point). It is therefore a general practice to denote a staphylococcus by its "phage pattern "-usually a list of the phages that lyse it strongly. Thus a staphylococcus reported as $3 \mathrm{~B} / 3 \mathrm{C} / 55$ was lysed strongly by these three phages; one reported as $6 / 7 / 47 / 53 / 54 / 75+$ was lysed strongly by these six phages and weakly by others (Fig. 9).

The number of different phage patterns observed is very large indeed, but some codification has been possible with the recognition of four principal lytic groups (e.g., Williams and Rippon, 1952; Williams, Rippon, and Dowsett, 1953; Rippon,
1956). Thus it was shown, for example, that phage 52 is very much more likely to be found in a lytic pattern with phages 29 and $52 \mathrm{~A}$ than with phages $3 \mathrm{~A}$ or 47 . Phages 29,52 , and $52 \mathrm{~A}$, with some others, thus constitute one distinct lytic group ; phage $3 \mathrm{C}$ is characteristic of a second, and phages 6 and 47 of a third, such group (see Table VIII). Further experience has confirmed the general usefulness of such groups, but has also emphasized that between 1 and $10 \%$ of strains are lysed by phages of both Groups I and III, even when phages are used at R.T.D., and that the number is considerably increased when the stronger phage filtrates are used.

In general it has not been difficult to assign the phages to one of the four groups, but some, e.g., phage 81 , are associated in pattern reactions with phages of both Groups I and III, and others, such as $47 \mathrm{~A}$, are usually the only phage lysing a strain and so cannot easily be grouped.

The idea that the three principal groups distinguished by phage typing (I, II, and III) had a real basis in the structure of the staphylococcus gained support from Hobbs' (1948) and Wahl and Fouace's (1952) reports that they correspond in a broad way to the three principal serological groups defined by Cowan (1939). That this is certainly no more than a broad correspondence is shown by Oeding's recent re-examination of a serological typing method (Oeding, 1953 ; Oeding and Vogelsang, 1954), and is to some extent implicit in Hobbs' (1948) earlier report. This aspect requires further investigation.

The general arrangement of the three principal groups was derived largely independently by Blair and Carr (1953), who also noted the occurrence of strains lysed by phages of the two groups now known as I and III. Jackson, Dowling, and Lepper (1954) also confirmed the segregation of patterns into three groups. 
In epidemiological investigations it is usually insufficient to classify the staphylococci into the three broad groups; a finer subdivision is necessary. To establish the validity of any finer subdivision it is essential to demonstrate that the individual pattern of a particular strain is stable. Williams and Rippon (1952) studied the variation observed in the typing results of sets of strains known or thought to be derived from a common parent and suggested that numerous distinct patterns could be validly recognized within the three groups.

Replicate typing of single strains held in vitro usually gives a practically identical phage susceptibility pattern on successive occasions. Similarly pairs of strains from the nose and lesion of patients with boils, or sets of strains from patients infected simultaneously in a food-poisoning epidemic, usually show no more than minor differences. In extensive or long-continued outbreaks of infection in a hospital ward, a greater degree of variation in pattern often occurs, and one is not infrequently in some doubt as to the relation of a few of the strains to the epidemic type. On the results of our earlier studies we generally regard two similar strains as different only if one is lysed strongly by two phages that fail altogether to lyse the second ; in making distinctions between patterns it is necessary to take into account some of the weak lytic reactions, and as these are not included in the reported phage pattern this may sometimes lead to confusion unless an explicit statement on the similarity or dissimilarity of the two strains is made. For example, the first four strains in the list below would all be regarded as probably of one type, although their reported patterns differed. The fifth strain, although showing an overlap in phage pattern, would be regarded as different.

\begin{tabular}{|c|c|c|c|c|c|c|c|c|}
\hline \multirow{2}{*}{ Strain } & \multicolumn{7}{|c|}{ Phage } & \multirow{2}{*}{ Reported Pattern } \\
\hline & \begin{tabular}{l|l}
6 & 7
\end{tabular} & $42 E$ & 47 & 53 & 54 & 75 & 77 & \\
\hline $\begin{array}{l}1 \\
2 \\
3 \\
4 \\
5\end{array}$ & $\begin{array}{l}++1++ \\
++1+ \\
++= \\
+-\overline{1} \\
++1\end{array}$ & \pm & $\begin{array}{l}++ \\
++ \\
++ \\
++ \\
+\end{array}$ & $\begin{array}{l}++ \\
++ \\
+t \\
++ \\
++\end{array}$ & $\begin{array}{l}+\overline{+} \\
\pm+ \\
++ \\
\pm\end{array}$ & $\begin{array}{l}+t \\
++ \\
\pm \\
\pm\end{array}$ & $\begin{array}{c} \pm \\
\pm \\
\pm \\
++\end{array} \mid$ & $\begin{array}{l}\text { 6 7 47 535475+ } \\
6475354+ \\
6475354+ \\
64753+ \\
65377\end{array}$ \\
\hline
\end{tabular}

This interpretation is based on studies of the variability observed in typing with filtrates at R.T.D. It may require modification when the results of tests with strong filtrates are considered, and it can never be regarded as more than a guide. Thus, in a particular hospital outbreak, the epidemic strain might be quite consistently lysed by, say, phages $6,7,47,53$, and 54 strongly and by 77 weakly; in this case one would suspect a strain lysed only by $6,47,53$, and 54 of being different, even though it differed from the others by only one strong reaction. If the apparent epidemic type shows a considerable degree of variation, a strain naturally has to show a considerable difference in pattern before being regarded as distinct. Moreover the occurrence with one of two strains of strong lysis by a phage of a different group, even though they are otherwise similar in pattern, is regarded as strong evidence for a real difference. This method of interpretation means that one can usually decide, in any particular episode, whether two or more strains are the same or different, but makes it difficult to construct a general list of types, or to say how many different types can be recognized.

To some extent the reliance that we place on individual phage patterns within the broad groups is confirmed by epidemiological studies of strains with peculiar pathogenic activity (see below), such as the association of type 71 with impetigo; that of various bovine strains with particular coagulase activities (Edwards and Rippon, in preparation); and that of certain strains in a hospital with particular antibiotic sensitivities (e.g., Alder, Gillespie, and Thompson, 1955).

Most of the evidence that the detailed phage patterns are useful labels for the strains comes, however, from epidemiological studies, which will be discussed below ; but it may be said directly that few of the published reports give sufficiently detailed information for independent assessment of the validity of the differences in phage patterns recorded.

The only other special study of the reproducibility of the phage typing method was that of Jackson et al. (1954), who reported substantially similar results to those of Williams and Rippon.

Wahl and Lapeyre-Mensignac (1950) proposed a more complex system of classification, in which different weight was attached to lysis by different phages; some phages were regarded as major and some as minor phages. Later Wahl and Fouace (1952) simplified the scheme somewhat and recognized five phage groups, I and II corresponding to our similarly numbered groups while III comprised phages $47,53,54,44,44 \mathrm{~A}, 6$, and 7 , and IV, regarded as a less specific group, 42B, 42C, 42D, 42E, $47 \mathrm{~A}, 47 \mathrm{~B}$, and $47 \mathrm{C}$; the fifth group comprised strains lysed only by the rather non-specific phage 68. Subdivisions within these groups were recognized. In actual practice it seems likely that Wahl's classification would not lead to greatly differing results from our own, but no reports seem to be 
available of any epidemiological investigations in which it has been tested. As we have noted already, it is on the results of epidemiological application that any scheme of typing stands or falls.

One particular criticism of the epidemiological use of phage typing needs comment: namely, Smith's suggestion that staphylococci may have their phage sensitivity pattern changed by acquiring a new phage derived from another staphylococcus growing in the same site. Smith (1948b) and Lowbury and Hood (1953) demonstrated that this might happen in vitro, and Smith produced suggestive evidence of its occurrence in nature. How important such change may be is not known, but our general experience with typing strains isolated over a period, often of months, from outbreaks of hospital infection suggests that it is not of major importance.

\section{Applications of Phage Typing}

Distribution of Phage-types.-It is fundamental to the use of any typing method in the epidemiological study of so ubiquitous an organism as Staph. aureus that we should know something of the relative frequency of various phage types in the population. The method by which the types are recorded makes an analysis of the frequency distribution of phage types difficult, but some indication of the numbers of different types was given by Williams and Rippon's observation that some 132 distinct patterns could be recognized among 259 independent strains from various sources (though not all of these patterns would be regarded as distinct by the convention given above), and that only three patterns were represented by more than 10 strains each; one (52A) had 39 strains.

In an investigation now in progress in a maternity hospital, we have tested 251 strains of staphylocosci from the noses of healthy babies, and from a number of septic lesions on the babies and their mothers. Only nine strains were insusceptible to all phages. The remaining 242 strains could be assigned to 33 different types on the basis of their phage patterns ; 14 of these types were represented by six or more strains and four of them by 20 or more strains (Table IX). The results demonstrated the importance of distinguishing the different patterns within the groups. For example, one Group I type was evidently spreading in epidemic fashion, being represented by 21 strains ; if all Group I strains had been regarded as of one type, one would have wrongly considered no fewer than 47 of them as belonging to the epidemic type.

More information is available on the distribution of staphylococci into the three principal phage
TABLE IX

RESULTS OF TYPING 251 STAPHYLOCOCCI FRCM A MATERNITY HOSPITAL

\begin{tabular}{|c|c|c|c|c|c|}
\hline \multicolumn{2}{|c|}{ Phage Group } & \multirow{2}{*}{$\mid \begin{array}{c}\text { No. of } \\
\text { Distinct } \\
\text { Phage } \\
\text { Patterns } \\
\text { (" Types") }\end{array}$} & \multirow{2}{*}{$\begin{array}{l}\text { No. of } \\
\text { Strains }\end{array}$} & \multicolumn{2}{|c|}{ No. of Types With } \\
\hline $\begin{array}{c}\text { On Basis of } \\
\text { Phages } \\
\text { at R.T.D. }\end{array}$ & $\begin{array}{c}\text { On Basis of } \\
\text { Strong } \\
\text { Phages }\end{array}$ & & & $\begin{array}{c}>20 \\
\text { Strains }\end{array}$ & $\begin{array}{c}>5 \\
\text { Strains }\end{array}$ \\
\hline $\begin{array}{l}\text { I } \\
\text { II } \\
\text { III } \\
\text { III } \\
\text { I/III } \\
\text { I/II/III } \\
-\end{array}$ & $\begin{array}{l}\text { I } \\
\text { II } \\
\text { III } \\
\text { I/III } \\
\text { I/III } \\
\text { I/IIIIII } \\
\text { I/III }\end{array}$ & $\begin{array}{r}10 \\
7 \\
11 \\
1 \\
2 \\
1 \\
1\end{array}$ & $\begin{array}{r}68 \\
49 \\
45 \\
3 \\
69 \\
1 \\
7\end{array}$ & $\begin{array}{l}1 \\
1 \\
1 \\
0 \\
1 \\
0 \\
0\end{array}$ & $\begin{array}{l}5 \\
3 \\
3 \\
0 \\
1 \\
1 \\
1\end{array}$ \\
\hline \multicolumn{2}{|c|}{ Untypable } & - & 9 & - & - \\
\hline \multicolumn{2}{|c|}{ Total } & 33 & 251 & 4 & 14 \\
\hline
\end{tabular}

groups. In a survey of normal persons and patients admitted to an isolation hospital with non-staphylococcal illness, Williams et al. (1953) found $24 \%$ of nasal carriers to have strains of Group I, 14\% Group II, and 18.5\% Group III (at the time Group IV was not separated, but Group IV strains are not in any case common in the human nose); $5 \%$ were of unclassifiable patterns and $38 \%$ untypable, that is resistant to all the phages employed at R.T.D. Staphylococci from the faeces or perianal swabs of normal people had a percentage distribution into the same groups of $8,10,30,3$, and 49 respectively. Rountree (1953) obtained similar figures for nasal carriers in Australia. Rountree and Barbour (1951) recognized 10 different patterns, representing all three groups, among 149 strains from the noses of healthy nurses.

\section{Differences between Human and Animal Staphylococci}

Early in the development of the phage typing method Macdonald (1946) found that 157 of 184 staphylococci isolated from milk and from cases of bovine mastitis were lysed by phage 42D (now allocated to Group IV). Later work confirmed the great predominance of such strains in the bovine udder and indicated that subtypes could be recognized (Smith, 1948c). Later Price, Neave, Rippon, and Williams (1954), studying a considerable number of herds of cows in various parts of S.E. England, found that, in addition to various Group IV strains, staphylococci of Group III were common. It was particularly noticeable that, in contrast to strains from human material, many of the bovine ${ }^{\circ}$ strains were very sensitive to the phages; strains lysed by phages of both Groups I and III were not uncommon, and some strains were lysed by practically all the phages used.

There is less information on staphylococci from other animals. Smith (1948c) found strains from 
sheep to be untypable with the set of phages received from Wilson and Atkinson. In later, unpublished, work at the Staphylococcal Reference Laboratory a small number of strains from sheep have been tested and found sensitive ; strains from dogs and poultry have also been typed. Apart from bovine strains, animal staphylococci are more often insensitive to the typing phages than human strains (Levy, Rippon, and Williams, 1953) ; this is not surprising since the phages were selected for testing human strains.

\section{Staphylococci with Peculiar Pathogenic Abilities}

It is gradually becoming clear that, for various reasons, particular phage types of staphylococci are more or less characteristic of some diseases.

Food-poisoning.-In early studies it was found that staphylococci producing outbreaks of enterotoxin food-poisoning were typically lysed by phages 6 and 47 (e.g., Allison, 1949; Allison, Hobbs, and Martin, 1949). Later work has shown that food-poisoning strains may have a great many other phage patterns, but virtually all of them belong to the broad Group III (Williams et al., 1953). In a more recent analysis (Williams, unpublished) of 93 well-authenticated food-poisoning incidents, 88 of the presumably causal strains of Staph. aureus proved to be typable: 77 were of Group III and six were of Group IV (42D) ; two were lysed by phages of both Groups I and III ; and there were two strains of Group II and one of Group I. Both the Group II strains were isolated from imported food that was apparently contaminated in the country of origin.

A further interesting point emerged from this analysis. There were six outbreaks of foodpoisoning due to imported canned food that appeared to have been contaminated before the can was opened by the consumer; and we have received 12 strains for typing from food-poisoning outbreaks in other countries. Three of the 18 strains were of Group II, which was not responsible for any of the food-poisoning incidents in which the contamination apparently arose within Britain.

As is well known, the laboratory testing of the staphylococcus enterotoxin is not satisfactorily established, and no one has yet carried out any extensive survey even using the present rather unsatisfactory tests, by monkey-feeding or intraperitoneal inoculat:on of kittens, to determine what proportion of all Group III strains are capable of producing enterotoxin. At present, therefore, although we can say that, from experience in
Britain, a staphylococcus that causes food-poisoning is almost certain to belong to phage Group III, we cannot judge the likelihood that any particular strain of a Group III staphylococcus produces enterotoxin.

Antibiotic Resistance.-Staphylococci resistant to penicillin first appeared in many numbers in 1946-47, and in 1949 Barber and Whitehead were able to show that some $60 \%$ of penicillin-resistant strains were lysed principally by phages 6,7 , or 47 (Group III) and $15 \%$ by $52 \mathrm{~A}$ (Group I). As resistant strains became more widespread, they were observed among all phage groups, and in 1953 Williams et al. found $17 \%$ to belong to Group I, $13 \%$ to Group II, $35 \%$ to Group III, and 35\% untypable with phages used at R.T.D. These findings are typical of those recorded in the literature (Table X). At present

TABLE $X$

DISTRIBUTION OF ANTIBIOTIC-RESISTANT STAPHYLOCOCCI INTO PRINCIPAL PHAGE GROUPS

\begin{tabular}{|c|c|c|c|c|c|c|c|}
\hline \multirow[b]{2}{*}{ Reference } & \multirow[b]{2}{*}{ Antibiotic } & \multirow[b]{2}{*}{$\begin{array}{c}\text { No. } \\
\text { of } \\
\text { Strains }\end{array}$} & \multicolumn{5}{|c|}{ Percentage } \\
\hline & & & 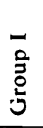 & 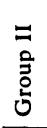 & 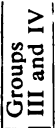 & 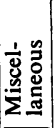 & $\begin{array}{l}\frac{0}{0} \\
\frac{0}{0} \\
\frac{0}{2} \\
\vdots \\
0\end{array}$ \\
\hline $\begin{array}{l}\text { Barber and Whitehead } \\
\text { (1949) }\end{array}$ & Penicillin & 140 & 16 & 7 & 63 & 一 & 21 \\
\hline $\begin{array}{l}\text { Rountree and Thomson } \\
\text { (1949) }\end{array}$ & "' & 121 & $?$ & $?$ & 75 & $?$ & 9 \\
\hline $\begin{array}{l}\text { Rountree and Thomson } \\
\text { (1952) }\end{array}$ & ," & 592 & $? 7$ & $? 2$ & 70 & $?$ & 19 \\
\hline Rountree (1953) & "* & 1,157 & 7 & 1 & 74 & 5 & 12 \\
\hline $\begin{array}{l}\text { Williams et al. (1953). } \\
\text { Barber et al. (1953) }\end{array}$ & , & $\begin{array}{l}104 \\
240\end{array}$ & $\begin{array}{l}17 \\
43\end{array}$ & $\begin{array}{r}13 \\
3\end{array}$ & $\begin{array}{l}35 \\
16\end{array}$ & $\begin{array}{l}0 \\
0\end{array}$ & $\begin{array}{l}35 \\
38\end{array}$ \\
\hline Fouace and Lutz (1953) & ," & 129 & 20 & 5 & 74 & $?$ & $?$ \\
\hline Jackson et al. (1954) . . & , & 195 & 10 & $<1$ & 62 & 28 & $\overline{10}$ \\
\hline Wallmark (1954) $\quad \ldots$ & , & 364 & $?$ & 3 & 65 & $?$ & 12 \\
\hline $\begin{array}{l}\text { Fusillo et al. (1954) } \\
\text { Knight and Holzer }\end{array}$ &., & $\begin{array}{l}362 \\
372\end{array}$ & $\begin{array}{l}3 \\
5\end{array}$ & $<\begin{array}{l}1 \\
0\end{array}$ & $\begin{array}{l}75 \\
92\end{array}$ & $\begin{array}{l}3 \\
0\end{array}$ & $\begin{array}{l}19 \\
13\end{array}$ \\
\hline$(1954)$ & & & & & & & \\
\hline $\begin{array}{l}\text { Barber and Burston } \\
\text { (1955) }\end{array}$ & " & 55 & 15 & 7 & 78 & 0 & 0 \\
\hline Parker et al. (1955) $\quad$. & ", & 149 & 29 & 15 & 25 & 3 & 28 \\
\hline Rountree et al. (1951) & Strepto- & $\begin{array}{r}326 \\
70\end{array}$ & $\begin{array}{r}43 \\
?\end{array}$ & $\begin{array}{l}2 \\
?\end{array}$ & $\begin{array}{l}45 \\
76\end{array}$ & $\begin{array}{l}6 \\
?\end{array}$ & $\begin{array}{l}4 \\
4\end{array}$ \\
\hline $\begin{array}{l}\text { Knight and Holzer } \\
(1954)\end{array}$ & , & 348 & 5 & $<1$ & 91 & 0 & 4 \\
\hline $\begin{array}{l}\text { Barber and Burston } \\
\text { (1955) }\end{array}$ & , & 30 & 0 & 0 & 100 & 0 & 0 \\
\hline $\begin{array}{l}\text { Alder et al. (1955) } \\
\text { Jackson et al. (1954) .. }\end{array}$ & Chlortet- & $\begin{array}{r}96 \\
147\end{array}$ & $\begin{array}{r}0 \\
16\end{array}$ & $\begin{array}{l}\mathbf{0} \\
\mathbf{0}\end{array}$ & $\begin{array}{l}98 \\
35\end{array}$ & $\begin{array}{r}1 \\
49\end{array}$ & 1 \\
\hline $\begin{array}{l}\text { Knight and Holzer } \\
\text { (1954) }\end{array}$ & $\begin{array}{l}\text { Tetracyc- } \\
\text { line }\end{array}$ & 335 & 2 & 0 & 95 & 0 & 3 \\
\hline $\begin{array}{l}\text { Barber and Burston } \\
\text { (1955) }\end{array}$ & $\begin{array}{l}\text { Oxytetra- } \\
\text { cycline }\end{array}$ & 27 & 0 & 0 & 100 & 0 & 0 \\
\hline Alder et al. (1955) . & Froth- & 37 & 3 & 0 & 97 & 0 & 0 \\
\hline & $\begin{array}{l}\text { Erytnro- } \\
\text { mycin }\end{array}$ & 33 & 0 & 0 & 91 & 9 & \\
\hline
\end{tabular}

(1) In several of Rountree's papers some or all of the types other than those of Group III are entered as " other types."

(2) Jackson et al. and Fouace and Lutz included no untypable

(3) Wallmark used a different series of phages from other workers, and had no representative of Group $I$.

(4) Staphylococci from lesions have been selected for inclusion in this table where possible. 
resistance to the broad-spectrum antibiotics appears to be largely confined to strains of Group III, though in this case the distribution is less well documented. These associations with antibiotic resistance mean that Group III strains have become the typical "hospital staphylococci."

In any particular institution there tends to be a fairly close relation between the antibiotic resistance of the strains and their phage pattern; the antibiotic resistance pattern can to some extent be used as a simple typing method, but, because the number of different resistance patterns is limited, and because staphylococci may sometimes alter in their resistance to the broad-spectrum antibiotics without altering phage type (e.g., Barber, 1949 ; Fairbrother, Parker, and Eaton, 1954; Wise, Voigt, Collin, and Cranny, 1955), phage typing is a much more reliable method.

Pneumonia.-During the influenza epidemics of 1949 and 1951 staphylococci from patients with fulminating pneumonia, often demonstrably postinfluenzal, were found to be significantly more often of Group I than were strains from healthy carriers or from patients with less severe pneumonia (Stuart-Harris, Franks, and Tyrrell, 1950 ; Williams et al., 1953). No further opportunity has arisen to attempt confirmation of these observations, but it may be noted that during the influenza B epidemic of 1954-55 staphylococci were received from a number of similar cases without any preponderance of Group I strains being observed (unpublished).

Impetigo.-Up to some $70 \%$ of staphylococci from acute cases of impetigo contagiosa have been found to be lysed solely by one phage 71 , and a further $6-10 \%$ are lysed by phage 71 and other phages of the Group II to which 71 belongs (Parker et al., 1955 ; Barrow, 1955 ; Spittlehouse, 1955). Confirmation of the existence of a specific type was obtained by the first-named authors from the special ability of impetigo staphylococci to inhibit the growth of $C$. diphtheriae mitis; the strains were also very commonly penicillinresistant.

Skin Sepsis.-Apart from the specific association of type 71 with impetigo there have been previous indications (Rountree, 1953 ; Roodyn, 1954) that strains causing boils and similar lesions tend to belong to Group II more than one might expect from the frequency of this group in the nose of normal people. Recently Alder et al. (1955) have found penicillin-resistant strains of Group I especially frequently responsible for furunculosis among the nurses in a hospital.
Although no explicit statistics are available, there appears to be an undue prevalence of strains of Group I $(52,52 / 52 \mathrm{~A}, 52 \mathrm{~A} / 79$ etc.) causing outbreaks of skin sepsis in maternity hospitals.

Enterocolitis.-From limited experience the staphylococci from patients with acute enterocolitis following antibiotic therapy appear to belong largely to Group III and they are often rather resistant to the typing phages.

\section{"Epianthropology"}

Numerous investigations have demonstrated the frequency with which staphylococcal infections in man are self-infections from normal carrier sites. Phage typing has played a large part in establishing this conclusion.

Carrier State.-Some $50 \%$ of normal adults carry Staph. aureus in the anterior nares and 20$40 \%$ carry the organism on the skin. The majority of carriers appear to harbour only one type in the nose (Williams, 1946; Rountree and Barbour, 1951), but two or more types are more commonly found on the skin; nevertheless, of 36 persons yielding typable strains from both nose and skin, 31 had strains of the same phage type in both sites. Phage typing has also shown that the persistent nasal carriers are usually constantly infected by a single type-often for quite long periods; transient carriers often carry different types in successive periods of carriage (Williams, 1946 ; Gould and McKillop, 1954).

Hospital personnel, especially nurses, are more frequently nasal carriers than persons of the same age and sex outside hospital (e.g., Miles, Williams, and Clayton-Cooper, 1945) ; in recent years it has been shown by phage typing that there is commonly one "hospital type" of staphylococcus, now usually penicillin-resistant, that predominates in any particular institution (e.g., Barber, 1949). Rountree and Barbour (1951) have shown that the nurses acquire the new strain soon after beginning work in the wards, and suggested that it was the $\tilde{O}$ nurses who were originally non-carriers who were the most likely to acquire it.

Hospital in-patients commonly acquire the hos- 0 pital staphylococcus, in the nose if not in a wound, and this is especially true of infants born in hos- ? pital (Barber, Hayhoe, and Whitehead, 1949; 궁 Barber, Wilson, Rippon, and Williams, 1953). 웅 Dowling, Lepper, and Jackson (1953) followed a number of carriers in their homes after discharge $\mathbb{Q}$ from hospital and showed that they spread the $\bar{\sigma}$ hospital type to their families to a very limited extent. 
Source of Infection in Boils and Other Septic Lesions.-Phage typing has shown that patients who suffer from recurrent boils and styes commonly yield a staphylococcus of the same type from each of their successive lesions (Roodyn, 1954 ; Valentine and Hall-Smith, 1952 ; Tulloch, 1954) and this type is also very common in the nose. Valentine and Hall-Smith were able to "cure" most of their patients with recurrent furunculosis by measures aimed particularly at eliminating the staphylococcus from the nose.

The phenomenon of self-infection of wounds with staphylococci from the patient's own carrier site was demonstrated for small wounds of the hand by Williams, Miles, Clayton-Cooper, and Moss (1949); for a septic lesion, seapox, of the hands of fishermen by Waage (1952) ; and for beatlesions in miners by Atkins and Marks (1952); in the latter case staphylococci of the infecting type were shown to be carried by some of the patients for several months before the onset of the septic condition.

\section{Epidemiology}

Hospital Infection.-Without doubt the most valuable contribution made by phage typing has been the unequivocal demonstration that hospital cross-infection with staphylococci is widespread and is in very large part responsible for the great prevalence of antibiotic-resistant strains infecting hospital patients. Unfortunately, many of the outbreaks have not been described in print, doubtless because of the difficulties of obtaining a clear-cut result in these investigations, so that this discussion has to rely rather heavily on the unpublished experience of the Staphylococcus Reference Laboratory.

Septic lesions, usually mild, have been very troublesome in maternity hospitals in Britain and elsewhere in the last two decades (e.g., Barber, 1949 ; Parker and Kennedy, 1949; Rountree and Freeman, 1955), and it is clear that the techniques ordinarily in use are unable to prevent the extensive spread of staphylococci. Babies born in hospital are almost all nasal carriers of Staph. aureus within a week of birth, and commonly one phage type of staphylococcus is predominant. Spread of the, temporarily, dominant type seems to occur even when clinical sepsis is rare (Barber et al., 1953): the dominant phage type seems to change from time to time and it may be that outbreaks of sepsis are due to a new invasion of the institution by a more virulent type.

Perhaps the only example of a widespread staphylococcal epidemic was provided by Rountree and Freeman's (1955) account of the spread of staphylococci of an apparently new type-80-to numerous maternity hospitals in Australia. The skin lesions that it produced were often severe. This type has also been observed recently in Britain, where several extensive hospital outbreaks have occurred.

In surgical wards similar problems have been revealed. Barber (1947) showed that when staphylococci in a wound apparently developed resistance to penicillin, they also changed in type ; subsequent experience showed that in particular hospitals there was a great tendency for the penicillinresistant staphylococci to have one phage pattern -and this pattern was different in different hospitals. It seemed clear that the development of resistance was in fact a manifestation of crossinfection. This conclusion still stands. Gould (1955) has claimed to have observed the development of penicillinase-producing variants in culture, with a correlated change of phage-type, but he has not given sufficient details to enable a critical assessment to be made ; even if his conclusion is accepted, the fact that hospital wards have their characteristic types indicates the paramount importance of cross-infection.

In our experience there are three types of epidemic that may be encountered, both among surgical wounds and in maternity units. In the first two all, or prastically all, the wound infections or skin lesions are infected with one phage type of staphylococcus.

In one type of outbreak only one or two carriers of the epidemic type can be found, and sometimes it is possible to demonstrate reasonably clearly that one of these is in fact responsible for the spread of infection. These epidemics are terminated by the exclusion of the responsible carrier : they are not, however, particularly common.

In the second type the epidemic type of staphylococcus is far more widespread-colonizing the noses of many of the staff, and being widely distributed in fomites (e.g., Blowers, Mason, Wallace, and Walton, 1955). Such epidemics cannot be terminated by the exclusion of a few carriers ; they may be controllable by extensive revision of aseptic technique, with, perhaps, the addition of local chemotherapy aimed at eliminating the staphylococcus from the noses of the carriers (Gould and Allan, 1954).

In the third type of epidemic, phage typing of strains from the lesions reveals a variety of types some or all of which are also represented among carriers on the staff : clearly there is no epidemic type and presumably no common source of infec- 
tion. Such epidemics are interpreted as resulting from a general lowering of aseptic standards.

It seems that, in the investigation of outbreaks of hospital infection, the first function of phage typing is to demonstrate whether an epidemic of either the first or second type exists, and this can only be done by typing staphylococci from a number of different patients' lesions. Only if one type of staphylococcus is present in several of them is it worth seeking carriers among the staff.

Although most outbreaks of infection offer relatively little difficulty in the interpretation of the typing results, it is certain that long-continued outbreaks, particularly those due to Group I strains, provide the greatest difficulties of deciding on the limits of variability allowed to the "epidemic type." From the fact that we have failed, by increasing the rigour of standardization of our technical methods, to reduce this variability to any great extent, we conclude that it probably indicates a real variation in the staphylococcus. But this implies that further search is needed for characteristics that show less variation.

Food Poisoning.-The investigation of outbreaks of food poisoning affords the most consistently elegant application of staphylococcal phage typing. As noted already the staphylococci responsible for toxin-food poisoning are, in Britain at least, practically all in phage Group III, and untypable strains are relatively rare. Presumably because the staphylococci isolated from food, patients, and food-handlers are, compared with those isolated from patients infected successively in an outbreak of hospital infection, separated from each other by relatively few generations, the reproducibility of the typing is better than in hospital outbreaks and difficulty in interpretation is rare.

On theoretical grounds it is desirable that an attempt should be made to isolate staphylococci from the patients' vomit, but this is often impracticable. It has been our experience that the isolation of staphylococci from faeces is generally satisfactory, but, since staphylococci are found in the faeces of some $15 \%$ of normal people, it is desirable that the same phage type should be recovered from two or more victims of the outbreak before concluding that the staphylococci are the cause of the illness. With this, and with the demonstration that the same phage type is present in the food, a search can be made for a source of the infection among food-handlers. It is perhaps worth repeating here the observation that phage typing cannot be used to determine whether or not a staphylococcus produces enterotoxin. If a staphylococcus is not of Group III it is very unlikely to produce the toxin, but within Group III some strains certainly do but others almost equally certainly do not produce it, and typing cannot distinguish those that do from those that do not.

\section{Comparison with Other Typing Methods}

It will be clear that the justification for the staphylococcus phage typing method is a purely pragmatic one : it is a useful method of labelling strains in order that their spread may be followed over what is usually a relatively short space of time.

We suffer from the fundamental difficulty that so little is known of the antigenic structure of the organism. This is not for want of searching. Cowan's early work (1939) showed that three broad groups could be recognized, but even at that level cross-reactions between Groups I and III were recognized. This work was extended by Christie and Keogh (1940) and by Hobbs (1948), who re-emphasized the technical difficulties. Although a number of new types were recognized no fundamental scheme of antigenic structure was discovered. It is curious, looking at this work in the light of the phage typing results, that so many of Hobbs' additional types belong to phage Group I or to the miscellaneous group. By phage typing the greatest variety of strains is encountered in Group III, although it has to be added that Group I strains often seem to show confusing minor differences and seem also to vary greatly in sensitivity to the typing phages.

More recently Mercier, Pillet, and Chabanier (1950) have claimed to have improved the method of production of agglutinating sera, and Oeding (e.g., 1954) has described a typing method based on the use of absorbed "single factor" sera. A definitive account of Oeding's methods has not been presented, and a great deal more epidemiological validation is required, but his analysis may well be a considerable advance.

Comparisons of the serological and phagetyping methods have been reported by Hobbs (1948), Wahl and Fouace (1952), and Oeding and Vogelsang (1954). There is general agreement that staphylococci of phage Groups I, II, and III tend to fall into the similarly numbered serological groups, but the tendency is certainly no more than a broad one. At the same time, Hobbs's study was made in the early days of phage typing, and there are some features in the work of Oeding and Vogelsang which suggest that a further comparison would be worth making.

The phage typing of staphylococci is clearly a less elegant method than the phage typing of the 
typhoid bacillus-partly no doubt because of our ignorance of the antigenic structure of the cocci, so that our different phages may react with quite different sorts of receptor on the cell; and probably partly also because, like the methods for $S$. paratyphi B and $S$. typhimurium, the typing phages are not all derived from one parent. It has not been possible to discover a systematic basis for type determination such as has resulted from the study of the type-determining phages of S. typhi.

No attempt has been made to type Staph. aureus by characterizing the phages carried by different strains, as used by Boyd (1950) for S. typhimurium, but the potential difficulties of such a method are well illustrated by Rippon's (1956) work on the classification of the phages.

A typing scheme has to be judged for its reliability by the extent to which strains known to originate from a common source are recognized as identical when isolated and tested on separate occasions ; and for its usefulness by its ability to distinguish between the various strains that are in circulation in the community. Whereas there are only a few hundred chronic carriers of $S$. typhi in the United Kingdom to-day, there must be at least $15,000,000$ staphylococcal carriers. It is therefore hardly surprising that we find a greater variety of staphylococci than of typhoid bacilli. This very profusion of the staphylococci makes the epidemiological assessment of their typing scheme immensely more difficult than is the assessment of the S. typhi scheme. However, typing of staphylococci is ordinarily needed for a study of local rather than widespread outbreaks of infection. It therefore seems worth attempting a fine distinction of the strains, even if some added complexity and uncertainty-because of the variation (which is almost certainly a real variation in the reaction of the staphylococci)-is thereby introduced. In fact the last 10 years' experience at the Staphylococcus Reference Laboratory has fully vindicated the acceptance of these complexities. It has become entirely clear that the method is ordinarily capable of answering all the important questions demanded of it in epidemiological studies of staphylococcal infections.

\section{REFERENCES \\ Staphylococcus aureus}

Alder, V. G., Gillespie, W. A., and Thompson, M. E. M. (1955). J. Path. Bact., 70, 503.

Allison, V. D. (1949). Proc. roy. Soc. Med., 42, 216

Hobbs, B. C., and Martin, P. H. (1949). Mon. Bull. Minist. Hlth

Atkins, J. B., and Marks, J. (1952). Brit. J. industr. Med., 9, 296.
Barber, M. (1947). J. Path. Bact., 59, 373.

(1949). J. gen. Microbiol., 3, 274.

and Burston, J. (1955). Lancet, 2, 578.

Hayhoe, F. G. J., and Whitehead, J. E. M. (1949). Ibid., 2, 1120 and Whitehead, J. E. M. (1949). Brit. med. J., 2, 565.

Wilson, B. D. R., Rippon, J. E., and Williams, R. E. O. (1953). J. Obstet. Gynaec. Brit. Emp., 60, 476.

Barrow, G. I. (1955). J. Hyg. (Lond.), 53, 495

Blair, J. E., and Carr, M. (1953). J. infect. Dis., 93, 1.

Blowers, R., Mason, G. A., Wallace, K. R., and Walion, M. (1955). Lancet, $2,786$.

Boyd, J. S. K. (1950). J. Path. Bact., 62, 501.

Burnet, F. M., and Lush, D. (1935). Ibid., 40, 455

Christie, R., and Keogh, E. V. (1940). Ibid., 51, 189.

Cowan, S. T. (1939). Ibid., 48, 169.

Dowling, H. F., Lepper, M. H., and Jackson, G. G. (1953). Amer. J. publ. Hith, 43, 860 .

Fairbrother, R. W., Parker, L., and Eaton, B. R. (1954). J. gen. Microbiol., 10, 309.

Farrant, J. L., and Rountree, P. M. (1953). Ibid., 9, 288.

Fisk, R. T. (1942). J. infect. Dis., 7 I, 153, 161.

- and Mordvin, O. E. (1944). Amer. J. Hyg., 4J, 232.

Fouace, J., and Lutz, A. (1953). Ann. Inst. Pasteur, 85, 387.

Fusillo, M. H., Roerig, R. N., Metzger, J. F., and Ernst, K. F (1954). Amer. J. publ. Hlth, 44, 317.

Goldberg, S. (1954). Science, 12v, 1041

Gould, J. C. (1955). Nature (Lond.), 176, 176. and Allan, W. S. A. (1954). Lancet, $2,988$.

and McKillop, E. J. (1954). J. Hyg. (Lond.), 52, 304

Hobbs, B. C. (1948). Ibid., 46, 222

Hood, A. M. (1953). Ibid., 51, 1.

Jackson, G. G., Dowling, H. F., and Lepper, M. H. (1954). J. Lab clin. Med., 44, 14, 29, 41.

Knight, V., and Holzer, A. R. (1954). J. clin. Invest., 33, 1190

Lowbury, E. J. L., and Hood, A. M. (1953). J. gen. Microbiol., 9, 524.

Levy, E., Rippon, J. E., and Williams, R. E. O. (1953). Ibid., 9, 97.

McClure, W. B., and Miller, A. M. (1946). Canad. med. Ass. J., 55, 36

Macdonald, A. (1946). Mon. Bull. Minist. Hlth (Lond.), 5, 230.

Mercier, P., Pillet, J., and Chabanier, P. (1950). Ann. Inst. Pasteur, $78,457$.

Miles, A. A., Williams, R. E. O., and Clayton-Cooper, B. (1944).

(1953). Acta path. microbiol. scand., 33, 324. (1954). Ibid., 3 t, 34

- and Vogelsang, T. M. (1954). Ibid., 34, 47.

Parker, M. T., and Kennedy, J. (1949). J. Hyg. (Lond.), 47, 213. Tomlinson, A. J. H., and Williams, R. E. O. (1955). Ibid., 53, 458.

Price, P., Neave, F. K., Rippon, J. E., and Williams, R. E. O. (1954). J. Dairy Res., 21, 342.

Rippon, J. E. (1954). Ph.D. Thesis: University of London.

(1956). J. Hyg. (Lond.). In the press.

Roodyn, L. (1954). Brit. med. J., 2, 1322.

Rountree, P. M. (1949a). J. gen. Microbiol., 3, 153.

- (1949b). Ibid., 3, 164.

(1953). Lancet, 1, 514.

and Barbour, R. G. H. (1951). J. Path. Bact., 63, 313. - and Thomson, E. F. (1951). Lancet, 1, 435.

and Freeman, B. M. (1955). Med. J. Aust., 2, 157.

and Thomson, E. F. (1949). Lancet, 2, 501 .

- (1952). lbid., 2, 262.

Smith, H. W. (1948a). Nature (Lond.), 161, 397.

(1948b). J. Hyg. (Lond.), 46, 74, 82 .

- (1948c). J. comp. Path., 58, 179.

Spi tlehouse, K. E. (1955). Lancet, $2,378$.

Stuart-Harris, C. H., Franks, Z., and Tyrrell, D. (1950). Brit. med. J., $1,263$.

Tulloch, L. G. (1954). Ibid., 2, 912.

Valentine, F. C. O., and Hall-Smith, S. P. (1952). Lancet, 2, 351.

Vogelsang, T. M. (1953). Acta path. microbiol. scand., 33, 435.

Waage, R. (1952). Nord. hyg. T., 33, 57.

Wahl, R., and Blum-Emerique, L. (1949). Ann. Inst. Pasteur, 77, 561.

- and Fouace, J. (1952). Ibid., 82, 54

Wa lapeyre-Mensignac, P. (1950). Ibid., 78, 353, 765

Wallmark, G. (1949). Nord. Med., 41, 806.

Will (1954). Acta path. microbiol. scand., 34, 57.

Williams, R. E. O. (1946). J. Path. Bact., 58, 259.

and Miles, A. A., Clayton-Cooper, B.,and Moss, B. (1949).

Spec. Rep. Ser. med. Res. Coun. (Lond.), No. 26.

- and Rippon, J. E. (1952). J. Hyg. (Lond.), 50, 320.

Williams, and Dowsett, L. M. (1953). Lancet, 1, 510.

Williams, S., and Timmins, C. (1938). Med. J. Aust., 2, 687.

Wilson, G. S., and Atkinson, J. D. (1945). Lancet, 1, 647. Wise, R. I., Voigt, A. E., Collin, M. V., and Cranny, C. L. (1955). 